\title{
Anwendungsszenarien und Technologiebewertung von digitalen Werkerassistenzsystemen in der Produktion - Ergebnisse einer Interview-Studie mit Experten aus der Wissenschaft, der Politik und der betrieblichen Praxis
}

\author{
Verena Simone Lore Blumberg ${ }^{1}$ (D) . Simone Kauffeld ${ }^{1}$ \\ Online publiziert: 5 . Februar 2020 \\ (c) Der/die Autor(en) 2020
}

\section{Zusammenfassung}

Die Einführung neuer digitaler Technologien wie digitale Werkerassistenzsysteme (z.B. Datenbrillen und Tablets) im Produktionsbereich führen zu Veränderungen der Arbeitswelt. Bestehende Modelle der Arbeitsgestaltung betrachten Technologien als Antezedenzien für die Arbeitsgestaltung. Die Theorie der sozialen Gestaltung von Technologien nimmt an, dass unterschiedliche Akteure Einfluss auf die Gestaltung und Verbreitung von Technologien im betrieblichen Kontext nehmen. Bisher ist kaum bekannt, welche Erwartungen unterschiedliche Akteure an die Anwendung und Gestaltung digitaler Werkerassistenzsysteme stellen. Ziel dieses Beitrags der Zeitschrift Gruppe. Interaktion. Organisation. (GIO) ist es, die Sichtweisen relevanter Akteure im Technologieentwicklungsprozess zu erfassen und basierende darauf Handlungsund Gestaltungsempfehlungen für den Einsatz digitaler Werkerassistenzsysteme in der betrieblichen Praxis zu geben. Hierfür wurden 76 halbstrukturierte Interviews mit Wissenschaftlern, politischen Akteuren und betrieblichen Praktikern durchgeführt und inhaltsanalytisch ausgewertet. Als Ergebnis dieser Studie ist ein Kategoriensystem für die Einteilung von Anwendungsmöglichkeiten digitaler Werkerassistenzsysteme in der Produktion entstanden, das einen Ausgangspunkt für betriebliche Entscheidungsprozesse bei der Technologieeinführung darstellen kann. Die identifizierten Chancen und Risiken beim Einsatz von digitalen Werkerassistenzsystemen stellen Ansatzpunkte für die Technologiegestaltung und die Technologieimplementierung im Unternehmen bereit.

Schlüsselwörter Digitalisierung · Digitale Werkerassistenzsysteme · Arbeitsgestaltung · Technologiemanagement · Qualitative Studie · Produktion

Verena Simone Lore Blumberg, M. Sc.

v.blumberg@tu-bs.de

Prof. Dr. Simone Kauffeld

s.kauffeld@tu-braunschweig.de

1 Lehrstuhl für Arbeits-, Organisations- und

Sozialpsychologie, Technische Universität Braunschweig,

Spielmannstr. 19, 38106 Braunschweig, Deutschland 


\title{
Application scenarios and technology assessment of smart wearables and mobile smart devices in industrial work-results of an interview study with experts from science, politics and industrial practice
}

\begin{abstract}
New opportunities of digitization (e.g. smart glasses, tablets) affect all areas of life and lead to noticeable changes in the world of work. Existing models of work design point towards technology as a relevant antecedent for task characteristics. Building on the concept of the social shaping of technology, we argue that the perspectives of relevant stakeholders must be identified in order to understand the future use of smart wearables and mobile smart devices as well as their effects on work design. This article in the journal Gruppe. Interaktion. Organisation. (GIO) aims to identify possibilities for the application of smart wearables and mobile smart devices in the industrial sector and also describes chances and risks attached to their respective usage. Hereby, the views of different expert groups with relevance for technology design and implementation were included. 76 semi-structured expert interviews were conducted and analyzed with content analysis to answer the research question. As a result, an order system for the classification of applications of smart wearables and mobile smart devices in production was created. The results also provide insight into the different perspectives of these expert groups. Furthermore, implications for the positive use of smart wearables and mobile smart devices in production are presented and discussed.
\end{abstract}

Keywords Digitization · Smart wearables · Work design · Technology assessment · Qualitative study · Industrial work

\section{Einleitung}

Die zunehmende Digitalisierung durchdringt alle Lebensbereiche und verändert auch die Arbeitswelt spürbar. Dabei werden beispielsweise Prozesse und Abläufe immer stärker automatisiert, Mitarbeitende arbeiten Hand in Hand mit Robotern oder werden von smarten Assistenzsystemen und künstlicher Intelligenz unterstützt, um nur einige der Veränderungen zu benennen. International werden diese Veränderungen unter dem Schlagwort digitalization diskutiert. Digitalization beschreibt die Veränderungen und Neustrukturierungen unterschiedlicher Lebensbereiche durch die Anwendung digitaler Technologien (Brennen und Kreiss 2016; Stolterman und Croon Fors 2004). Im deutschsprachigen Raum hat sich für die konsequente Anwendung und Verbreitung neuer Technologien (z.B. Robotik, Informations- und Kommunikationstechnologien) im industriellen Produktionsumfeld der Begriff Industrie 4.0 durchgesetzt (Hirsch-Kreinsen 2016). Zielbild in der Industrie 4.0 ist die sog. Smart Factory, eine Fabrik mit erheblich gesteigerter Effizienz, in der die Komplexität auch bei kundenindividuellen Produkten beherrschbar ist und Störungen minimiert sind (Kagermann et al. 2013). Ermöglicht wird dies durch eine intelligente, sensorgestützte Vernetzung aller Akteure, also von Gütern und Maschinen, ebenso wie von Menschen (Hirsch-Kreinsen 2014; Kagermann et al. 2013; Spath et al. 2013). Die Einbindung des Menschen in die Smart Factory kann durch digitale Werkerassistenzsysteme (DWAS), wie Datenbrillen und Tablets, realisiert werden.

Durch diese neue Qualität und Allgegenwärtigkeit von Technologien in Arbeitsprozessen erhält auch die Diskussion über das Verhältnis und die wechselseitige Beeinflussung von Technologie und Arbeit neue Aufmerksamkeit (Cascio und Montealegre 2016; Parker und Grote 2019). Während in vielen Modellen der Arbeitsgestaltung Technologien als gegebene Kontextfaktoren angesehen werden, die die Anpassung von Arbeit und Mitarbeitenden nach sich ziehen, richten wir in unserem Beitrag den Blick auf Gestaltungsentscheidungen, die im Technologieeinführungsprozess von unterschiedlichen Akteuren getroffen werden. Damit folgen wir dem Aufruf von Parker und Grote (2019), den Antezedenzien von Arbeitsgestaltung mehr Aufmerksamkeit zu schenken. Ein Kernpunkt dieser Auseinandersetzung kann das Erfassen der Sichtweisen unterschiedlicher Akteure der Technologiegestaltung sein, um daraus Erkenntnisse über Antezedenzien der Arbeitsgestaltung ableiten zu können.

Die vorliegende Studie leistet einen Beitrag zum Forschungsbereich der Arbeitsgestaltung, indem sie die Sichtweisen unterschiedlicher Akteure erfasst und gegenüberstellt, die an der Gestaltung, Implementierung und Nutzung der neuen Technologien beteiligt sind. Dabei nutzen wir die Sichtweisen unterschiedlicher Akteure zu Anwendungsmöglichkeiten sowie erwarteten Chancen und Risiken beim Einsatz von DWAS für die Produktionsarbeit.

\subsection{Die Relevanz von Technologie für die Arbeitsgestaltung}

Die Arbeitsgestaltung versucht mit technischen, organisatorischen und ergonomischen Maßnahmen Arbeitsprozesse effektiv, produktiv und menschengerecht zu gestalten. Dabei werden sowohl unternehmensbezogene Ziele (z. B. Kostenziele, Qualitätsziele) als auch mitarbeiterbezogene Ziele (z. B. Ausführbarkeit, Persönlichkeitsförderlichkeit) ver- 
folgt. Beide Zielarten sind nicht immer miteinander vereinbar und verhalten sich teilweise konfliktär (Schaper 2019).

Ein grundlegendes Modell der Arbeitsgestaltung ist das Job Characteristics Model (Hackman und Oldham 1976). Das Modell beschreibt fünf zentrale Tätigkeitsmerkmale (Anforderungsvielfalt, Ganzheitlichkeit, Wichtigkeit, Autonomie und Rückmeldung), die Einfluss auf psychologische Erlebniszustände (Bedeutsamkeit der Arbeit, Verantwortung für Arbeitsergebnisse, Kenntnis über Arbeitsergebnisse) haben. Die Tätigkeitsmerkmale wirken über die psychologischen Erlebniszustände auf mitarbeiter- und arbeitsbezogene Outcomes. Eine hohe Ausprägung der zentralen Tätigkeitsmerkmale wird mit einer höheren Motivation der Mitarbeitenden, einer höheren Arbeitszufriedenheit und einer besseren Performance verbunden. Ansatzpunkte für die Arbeitsgestaltung werden in der Beeinflussung der gestaltbaren Tätigkeitsmerkmale gesehen. Interpersonelle, technologische oder situationsbezogene Faktoren, die ebenfalls Einfluss auf die Arbeitsgestaltung haben können, werden in dem Modell nicht berücksichtigt (Hackman und Oldham 1976).

Ein Modell der Arbeitsgestaltung, das explizit auch technologische Komponenten berücksichtigt, ist die soziotechnische Systemtheorie (Trist und Bamforth 1951). Die Theorie beschreibt, dass Arbeitssysteme aus einem sozialen System (z.B. Mitarbeitende, Rollen) und einem technischen System (z. B. Arbeitsprozesse, Technologien) bestehen und verweist darauf, dass sich die Systeme wechselseitig beeinflussen. Nur durch eine gemeinsame Optimierung beider Teilsysteme ist eine Verbesserung des Gesamtsystems und damit der Outcomes (z. B. Produktivität, Arbeitszufriedenheit) zu erreichen (Trist 1981).

Neuere Konzepte wie das erweiterte Modell der Arbeitsgestaltung (Parker et al. 2001) und das Work Design Framework (Morgeson und Campion 2003) integrieren beide Sichtweisen. Sie betrachten zusätzlich zu den Tätigkeitsmerkmalen auch soziale, individuelle, strukturelle und organisationale Einflussfaktoren als Antezedenzien der Tätigkeitsmerkmale. Sowohl die generell verfügbaren Technologien, als auch die genutzten Technologien werden als relevante Antezedenzien für die Arbeitsgestaltung benannt. Studien zum Einfluss von Technologien auf Tätigkeitsmerkmale zeigen uneinheitliche Ergebnisse. Während einige Studien von einer Verbesserung der Autonomie bei Einführung einer neuen Technologie berichten (z.B. Wall et al. 1990), kommen andere Studien zu dem Ergebnis, dass die Einführung neuer Technologien mit einer Verschlechterung der Autonomie einhergeht (z.B. Amick und Celentano 1991). Die Ergebnisse zeigen, dass die Effekte von Technologien auf die Arbeitsgestaltung nicht determiniert sind, sondern Technologien sowohl einen limitierenden als auch förderlichen Faktor für die Arbeitsgestaltung darstellen können (Parker et al. 2001; Parker und Wall
2001). Die Effekte hängen in einem erheblichen Maß von Faktoren wie der konkreten Gestaltung der Technologie und dem Technologieeinführungsprozess ab (Parker und Grote 2019; Waschull et al. 2020). Da Unternehmen bei der Einführung neuer Technologien aktiv Entscheidungen treffen und daraus Implikationen für die Arbeitsgestaltung entstehen, möchten wir nachfolgend auf das Technologiemanagement eingehen, das den Weg einer Technologie in die betriebliche Anwendung beschreibt.

\subsection{Technologiemanagement in Unternehmen}

Damit die richtigen Technologien nutzbringend für Unternehmen eingesetzt werden können, kommt dem Technologiemanagement eine große Bedeutung zu. Dieses beschäftigt sich mit der frühzeitigen Identifizierung, Gestaltung, Optimierung, Implementierung und Bewertung neuer Technologien im Unternehmen (Bullinger 1994). Schuh et al. (2011) erstellten auf Basis verschiedener Definitionen von Technologiemanagement ein Prozessmodell aus sechs grundlegenden, miteinander vernetzten Aktivitäten. Das Modell ist in Abb. 1 dargestellt. Ausgangspunkt ist die Technologiefrüherkennung, in der relevante Technologien identifiziert und Informationen gesammelt und analysiert werden. Dies bildet die Grundlage für strategische Technologieentscheidungen und die Technologieplanung. Anschließend werden Maßnahmen zur Technologieentwicklung, -verwertung und zum Technologieschutz erstellt und realisiert. Schuh et al. (2011) betonen, dass über alle Phasen hinweg die Technologiebewertung (oder auch Technikbewertung) eine entscheidende Funktion einnimmt und die Basis für Entwicklungs-, Einführungs- und Nutzungsentscheidungen für neue Technologien bildet.

Technikbewertung ist ein systematisches Vorgehen, mit dem der aktuelle Stand von Technik dargestellt, Entwicklungsmöglichkeiten erfasst und unmittelbare und mittelbare Folgen abgeschätzt werden. Ziel ist es Handlungs- und Gestaltungsmöglichkeiten für Technologien abzuleiten. Als Unterstützung bei der Bewertung von Technologien kann die VDI-Richtlinie 3780 zur Technikbewertung (VDI - Verein Deutscher Ingenieure 2000) genutzt werden. Bei der Betrachtung der Technikfolgen sollen neben technischen auch ,[...] wirtschaftliche, gesundheitliche, ökologische, humane, soziale und andere Folgen [...]" (VDI - Verein Deutscher Ingenieure 2000, S. 3) betrachtet werden. Diese breite Betrachtung von Technikfolgen unterstützt Forderungen aus der Forschung, dass bei der Diskussion über die Nutzung neuer Technologien im Produktionsumfeld neben der technischen Machbarkeit auch die Auswirkungen auf die Organisation und die Beschäftigten betrachtet und eine bewusste Gestaltung der neu entstehenden Mensch-MaschineSchnittstellen vorgenommen werden sollen (Hirsch-Kreinsen 2014; Ittermann et al. 2016). 
Abb. 1 Prozessmodells des Technologiemanagements mit den von Schuh et al. (2011) beschriebenen Aktivitäten. (Eigene Darstellung)

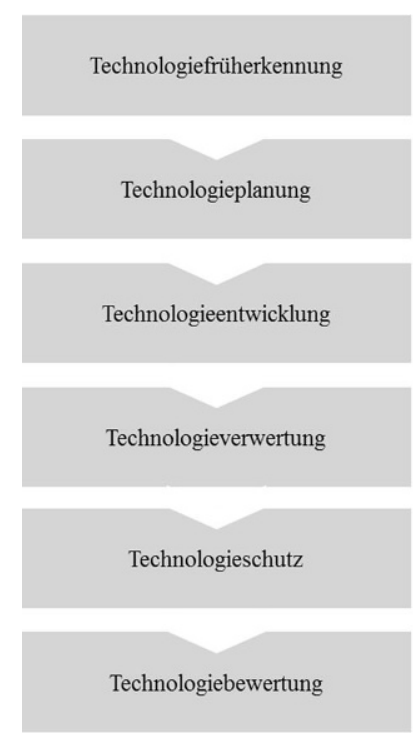

\subsection{Akteure bei der Technologiegestaltung und -einführung}

Während der technologische Determinismus beschreibt, dass es sich bei Technologien um gegebene, kaum veränderbare Artefakte handelt, die eine Veränderung und Anpassung der Gesellschaft und des Menschen nach sich ziehen, vertritt die Theorie der sozialen Gestaltung von Technologien (social shaping of technology) die Sichtweise, dass Technologien bewusst gestaltet werden können (Rammert 2016; MacKenzie und Wajcman 1999; Russell und Williams 2002). Grundannahme dieser Theorie ist, dass nicht nur die Technologie selbst, sondern auch ihre zentralen Eigenschaften, Nutzungsszenarien und konkrete Anwendungen eine Folge sozialer Prozesse und Entscheidungen sind (Russell und Williams 2002). Unterschiedliche Gruppen und Akteure nehmen durch das Treffen (bewusster und unbewusster) Entscheidungen Einfluss auf die Gestaltung und Verbreitung von Technologien (Williams und Edge 1996). Subjektive Einstellungen hinsichtlich der neuen Technologie, erwünschte Zielzustände oder zu vermeidende Risiken stellen daher bereits in der Technologieentwicklung und -implementierung bedeutsame Entscheidungsfaktoren dar.

Bei der Entwicklung und Einführung neuer Technologien in der Industrie 4.0 verweist Hirsch-Kreinsen (2016) unter anderem auf die Rolle der Wissenschaft (vorwiegend auf Informatikwissenschaften), die Rolle innovationspolitischer Akteure (z.B. Bundesministerium für Bildung und Forschung) und die Rolle der Unternehmensvertreter. Im betrieblichen Umfeld spielen neben Entscheidungsträgern auch Fachgruppen und Anwender eine bedeutsame Rolle (Russell und Williams 2002; Williams und Edge 1996). Die gerade benannten Gruppen können in unterschiedlicher
Identifikation relevanter Technologien und Bereitstellung relevanter Informationen und Analysen als Grundlage für strategische Technologienentscheidungen des Unternehmens

Konkrete Ausplanung der Anwendung von Technologien im Unternehmen

Realisierung der getroffenen Technologieentscheidungen durch Entwicklung neuer oder Optimierung bereits im Unternehmen vorhandener Technologien

Verwertung von Technologien zur Verbesserung unternehmensinterner Prozesse (z. B Produktionsprozesse) oder Verwendung von Technologien für Produkte des Unternehmens

Aktivitäten zum Schutz des unternehmensinternen, technologischen Wissens /KnowHows zum Erhalt von Wettbewerbsvorteilen

Überprüfung und Bewertung der Technologien hinsichtlich des Erfüllungsgrades bezogen auf an die Technologie gestellte Anforderungen und Ziele

Weise Einfluss auf die Technologiegestaltung, -anwendung und -verbreitung nehmen (siehe Tab. 1). Betriebsinterne Akteure wie Entscheider, Betriebsräte und Anwender können durch die Auswahl bestimmter Technologien, die bewusste Ausgestaltung und Reglementierung von Anwendungsmöglichkeiten sowie die Nutzung bzw. Nicht-Nutzung der Technologien die Entwicklung und Verbreitung beeinflussen.

Die Unterstützung oder Ablehnung neuer Technologien im betrieblichen Kontext wird auch im Promotorenmodell von Witte (1973) thematisiert. Das Modell beschreibt, dass betriebliche Akteure bei der Einführung neuer Technologien als Opponent Widerstand gegen die Neuerungen leisten können oder als Innovationsförderer (sog. Promotoren) die Einführung und Nutzung neuer Technologien aktiv voranbringen können. Widerstände können aufgrund von fehlendem Wissen (Nicht-Wissen), persönlichen oder motivationalen Gründen (Nicht-Wollen) oder betrieblichen Beharrungstendenzen (administrative Barrieren) entstehen und dazu führen, dass Veränderungsprozesse verhindert, verzögert oder verformt (z.B. Umsetzung von Alternativen) werden (Hauschildt und Salomo 2008). Promotoren tragen zur Überwindung und Lösung der Widerständen bei, indem sie Wissen einbringen (Fachpromotor), formelle Einflussmöglichkeiten ausüben (Machtpromotor) oder diplomatische Fähigkeiten einsetzen (Prozesspromoter) (Witte 1973; Hauschildt und Chakrabarti 1988). Im angelsächsischen Raum wurde ein ähnliches Konzept entwickelt, das die unterschiedlichen Promotorenrollen in einer Person, dem Champion, vereint (Howell und Higgins 1990). Ein Champion zeichnet sich durch ein hohes Maß an Zuversicht in den Erfolg der Innovation und Widerstandsfähigkeit bei Schwierigkeiten aus und sorgt dafür, dass die richtigen Personen im Innovationsprozess mitwirken (Howell et al. 
Tab. 1 Übersicht über die Expertengruppen inkl. Relevanz, Beschreibung und Wege der Identifikation geeigneter Experten sowie Anzahl der befragten Experten je Gruppe

\begin{tabular}{|c|c|c|c|c|}
\hline $\begin{array}{l}\text { Experten- } \\
\text { gruppe }\end{array}$ & Relevanz der Expertengruppe & Beschreibung der Expertengruppe & Identifikation der Experten & $\begin{array}{l}\text { Anzahl } \\
\text { befragter } \\
\text { Experten }\end{array}$ \\
\hline \multirow[t]{3}{*}{$\begin{array}{l}\text { Wissen- } \\
\text { schaft }\end{array}$} & $\begin{array}{l}\text { Externe Expertise (vgl. Froschauer } \\
\text { und Lueger 2003), fundiertes theo- } \\
\text { retisches Wissen in den betrachteten } \\
\text { Themenfeldern }\end{array}$ & $\begin{array}{l}\text { Mitarbeitende von Universitäten, } \\
\text { Fachhochschulen sowie }\end{array}$ & $\begin{array}{l}\text { Sichtung von Tagungsbänden und } \\
\text { Teilnehmenden von Fachkonfe- } \\
\text { renzen zum Thema Industrie 4.0, } \\
\text { Arbeit der Zukunft }\end{array}$ & $n=16$ \\
\hline & $\begin{array}{l}\text { Rolle der Wissenschaft bei der } \\
\text { Technikentwicklung (Rammert 2016) }\end{array}$ & Forschungseinrichtungen & $\begin{array}{l}\text { Durchsicht von Hochschul- und } \\
\text { Institutswebseiten }\end{array}$ & \\
\hline & $\begin{array}{l}\text { z. B. bezogen auf } \\
\text { Anwendungsmöglichkeiten oder } \\
\text { Einsatzgebiete für Technologien }\end{array}$ & $\begin{array}{l}\text { Autoren/Autorinnen von Fachar- } \\
\text { tikeln zum Thema Industrie 4.0, } \\
\text { Arbeit der Zukunft, Produktions- } \\
\text { arbeit, Digitalisierung, digitale }\end{array}$ & $\begin{array}{l}\text { Nutzung bestehender } \\
\text { Hochschulkontakte z. B. im } \\
\text { Rahmen von } \\
\text { Forschungskooperationen }\end{array}$ & \\
\hline
\end{tabular}

Werkerassistenzsystem digitale

Einbezug von Experten mit unterschiedlichen fachlichen Hintergründen (z. B. Arbeitswissenschaften, Fertigungstechnik, Unternehmensforschung, Informatik)

Politische Schaffung und Gestaltung von RahAkteure menbedingungen, z. B. Regelungen und Gesetzen (Rammert 1993) Anstoß von Diskussionsprozessen zu Innovationen und Technologien und Priorisierung der Themen in der Forschungs-, Innovations- und Bildungspolitik (Beyer et al. 2019)

\begin{tabular}{ll}
\hline Planer & Identifikation relevanter Technolo \\
& $\begin{array}{l}\text { Implementierung und Evaluation } \\
\text { neuer Technologien im } \\
\text { Unternehmenseinsatz }\end{array}$
\end{tabular}

neuer Technologien im

Mitarbeitende von

Bundesministerien, Vereinen und

Verbänden sowie von

Gewerkschaften, die sich mit den

Themen Industrie 4.0,

Digitalisierung, Produktionsarbeit,

Arbeitsgestaltung oder digitale

Werkerassistenzsysteme beschäftigen

Unternehmensinterne

Mitarbeitende aus dem indirekten

Bereich, die sich mit der

Einführung neuer Technologien

oder der Begleitung von

Pilotprojekten zu DWAS oder der

Arbeitsgestaltung in der

Produktion beschäftigen

Durchsicht von Webseiten $\quad n=14$

Empfehlung von Kooperationsstellen

Nutzung bestehender Kontakte zu

Verbänden z. B. im Rahmen von

Industrie 4.0-Projekten

Durchsicht von unternehmensin-

ternen Gremienberichten (Teilneh-

mer)

Identifikation von Mitarbeitenden

mit Beschäftigungsschwerpunkten

wie Industrie 4.0, Digitalisierung,

Werkerassistenzsysteme sowie

Industrial Engineering oder Tech-

nologiescouting

Empfehlung von Kollegen

Unternehmensinterne Betriebsräte und Betriebsratsreferenten, die sich mit den Themen

Arbeitsgestaltung, Digitalisierung, Industrie 4.0 oder Veränderung von Arbeit beschäftigen

Direkte Ansprache von Betriebsrä-

$n=14$ ten

Durchsicht unternehmensinterner

Gremienberichte (Teilnehmer)

Empfehlung von Kollegen

Unterrichtungs- und Beratungsrechte

(§ 90) bei der Gestaltung von Arbeitsplätzen und Arbeitsabläufen

Anwender Hohes Wissen über die konkrete Arbeitsaufgabe in der Produktion und die aktuell vorherrschende Arbeitsgestaltung

Einflussnahme auf die

Technologiegestaltung und durch bewusste Nutzungsentscheidungen auf die Anwendung technologischer Innovationen im betrieblichen Kontext (Rammert 1993, 2016)

\author{
Unternehmensinterne Mitarbeiten- \\ de aus der Produktion (Gewerke \\ Fertigung, Montage und Logistik) \\ Ansprache über Vorgesetzte \\ Teilweise mit Erfahrungen zum \\ Einsatz digitaler \\ Werkerassistenzsysteme aus \\ Ansprache über den Betriebsrat \\ Empfehlung von Kollegen
}

$n=15$
Pilotprojekten 
2005). Folkerts und Hauschildt (2001) konnten zeigen, dass Opponenten durch den Einbezug in den Veränderungsprozess und durch eine intensivere Auseinandersetzung mit den Inhalten der Veränderung zu Promotoren der Veränderung werden können. Weiterhin konnte gezeigt werden, dass Führungskräfte als Machtpromotoren einen direkten Einfluss auf die Entscheidung von Mitarbeitenden zur Nutzung neuer Technologien haben (Wieseke et al. 2010). Mit Ausnahme der Rolle des Machtpromotors sind die Rollen nicht an hierarchische Positionen innerhalb des Unternehmens gebunden und können daher von allen Mitarbeitenden eingenommen werden.

Außerhalb des Unternehmens schaffen Wissenschaftler durch die Entwicklung von Innovationen und deren Erforschung Grundlagenwissen. Durch fundierte Technikbewertungen und entsprechende Informationsbereitstellung schaffen Wissenschaftler ein Bewusstsein für Entwicklungsrichtungen, mögliche Chancen und Risiken der Technologien, Perspektiven relevanter Stakeholder sowie Herausforderungen und Optionen für die politische Gestaltung (Hennen et al. 2004). Wissenschaftliche Forschungsergebnisse können somit eine Grundlage für politische Meinungsbildung und Entscheidungsprozesse sein und zur Setzung von Rahmenbedingungen (z.B. Regelungen und Gesetzen) beitragen. Die unterschiedlichen Bewertungen von Technologien durch die unterschiedlichen Akteure, beispielsweise hinsichtlich möglicher Chancen und Risiken beim Technologieeinsatz, bilden die Grundlage für Verhandlungen im Technologieentwicklungs- und Technologieimplementierungsprozess (Jørgensen et al. 2009).

\subsection{Digitale Werkerassistenzsysteme und bisherige Erkenntnisse zu Anwendungsmöglichkeiten in der Produktion}

In der Smart Factory sind alle Güter, Maschinen und Menschen miteinander vernetzt (Hirsch-Kreinsen 2014; Kagermann et al. 2013; Spath et al. 2013). Mitarbeitende können durch DWAS in diese vernetzte Welt integriert werden. DWAS sind definiert als ,,[...] mobile oder körpernah tragbare Endgeräte (Wearables) in industrieller Anwendung [...]“" (Niehaus 2017, S. 5) und unterstützen Mitarbeitende zum Beispiel durch die kontextsensitive Bereitstellung relevanter Informationen. Vor allem im Produktionsumfeld bestehen beim Einsatz von DWAS große ökonomische Potenziale zur Effizienzsteigerung, Optimierung von Prozessen und Datenanalyse (McKinsey Global Institute 2015). Gleichzeitig ermöglichen DWAS die Beherrschung der stetig steigenden Komplexität der Produktionsarbeit durch die Fertigung kundenindividueller Produkte. Trotz der hohen Verbreitung von Smartphones, Tablets und zunehmend auch Datenbrillen im Consumer-Bereich erfolgt die Anwendung dieser Systeme im industriellen Umfeld eher langsam. Diesen Eindruck un- terstützen auch Evers et al. (2018), die berichten, dass sich DWAS immer noch in einer „Design- und Bereitstellungsphase" (S. 7) befinden.

Die geringe Verbreitung von DWAS in der betrieblichen Praxis kann ein Grund dafür sein, dass Studien zu DWAS bisher häufig praxisfern durchgeführt werden und nur einzelne Anwendungsmöglichkeiten betrachten (Niemöller et al. 2017). Eine übergeordnete Systematisierung von Nutzungsmöglichkeiten für DWAS ist derzeit nicht vorhanden. Dennoch bemühen sich einige Studien um eine Zusammenfassung identifizierter Anwendungsmöglichkeiten (Aleksy et al. 2011; Damiani et al. 2018; Evers et al. 2018; Niehaus 2017; Niemöller et al. 2017). In vier der fünf benannten Studien werden die Anleitung von Arbeitsaufgaben, die Instandhaltung und das Erlernen von Arbeitstätigkeiten bzw. die Anwendung von DWAS beim Training beschrieben. In drei der fünf Studien werden Anwendungsmöglichkeiten bei der Qualitätssicherung, der Dokumentation sowie der Anwendung für die Kollaboration und Kommunikation zwischen Mitarbeitenden angegeben. Obwohl in den einzelnen Studien unterschiedliche Technologien betrachtet wurden (z.B. untersuchten Aleksy et al. (2011) die Anwendung von tragbaren Computergeräten, wohingegen Damiani et al. (2018) AR- und VR-Anwendungen untersuchten), überschneiden sich die identifizierten Anwendungsmöglichkeiten für DWAS.

Ein Kritikpunkt an bisherigen Studien zur Identifizierung von Anwendungsmöglichkeiten von DWAS ist, dass diese häufig weder branchen- noch tätigkeitsspezifisch (Herterich et al. 2015) sind. Niemöller et al. (2017) begegnen diesem Kritikpunkt und betrachten in einer explorativen Fallstudie die Anwendung von Datenbrillen in der Logistikbranche. Sie identifizierten 36 Anwendungsfälle von Datenbrillen in der Logistik und erstellten eine erste Systematisierung, indem sie die Anwendungsfälle anhand der Prozessschritte in der Logistik (Prozessgruppe) und der spezifischen Funktion, die durch die Datenbrille zur Verfügung gestellt wird (Funktionsgruppe), organisierten. Als Ergebnis wurden die drei Prozessgruppen Management, Kommunikation und Value Added Services/Qualitätssicherung identifiziert. Die Funktionen der Datenbrille wurden mit Monitoring, Steuerung, Anleitung, Abruf von Videos, automatisierte Kontrolle, Identifizierung, Navigation und Sicherheit beschrieben.

Als Resümee zeigt sich, dass in den vorgestellten Studien unabhängig von der betrachteten Einzeltechnologie ähnliche Anwendungsfelder identifiziert wurden. Diesem Befund folgend, verzichten wir in dieser Studie auf eine detaillierte Unterscheidung einzelner Technologien und wählen den Begriff DWAS als Sammelbezeichnung unterschiedlicher Technologien, die den Mitarbeitenden im Produktionsprozess kognitiv unterstützen. Eine übergreifende Systematik zu Nutzungsmöglichkeiten von DWAS in der 
Produktion liegt unseres Wissens nach zum gegenwärtigen Zeitpunkt nicht vor. Weiterhin gibt es bisher keine verlässliche Datenlage dazu, wie DWAS in der betrieblichen Praxis angewendet werden (Aleksy et al. 2011; Niehaus 2017).

\subsection{Ziele der Studie und Forschungsfragen}

Ziel dieser Studie ist es, die Sichtweisen relevanter Akteure im Technologiegestaltungsprozess zu erfassen, um Handlungs- und Gestaltungsempfehlungen für die Technologien und deren Nutzung im Unternehmenskontext abzuleiten. Hierfür wird im ersten Schritt eine Systematik von Anwendungsmöglichkeiten von DWAS im Produktionsbereich, erstellt. Zur Ableitung der Handlungs- und Gestaltungsempfehlungen werden außerdem die erwarteten mittelbaren und unmittelbaren Chancen und Risiken des Technologieeinsatzes erfasst. Im Rahmen der Studie sollen die folgenden Forschungsfragen beantwortet werden:

Forschungsfrage 1 Welche Anwendungsmöglichkeiten bestehen aus Sicht unterschiedlicher Akteure/Expertengruppen für die Anwendung von DWAS in der Produktion (in den Gewerken Fertigung, Montage und Logistik)?

Forschungsfrage 2 Welche Chancen und Risiken bestehen aus Sicht unterschiedlicher Akteure/Expertengruppen bei der Anwendung von DWAS in der Produktion (in den Gewerken Fertigung, Montage und Logistik)?

\section{Methode}

In diesem Abschnitt werden die Datengewinnung und die Stichprobe vorgestellt. Weiterhin werden das Vorgehen bei der Datenaufbereitung und bei der Auswertung der qualitativen Daten beschrieben.

\subsection{Beschreibung der Stichprobe}

Um Anwendungsmöglichkeiten sowie Chancen und Risiken beim Einsatz von DWAS aus der Sicht unterschiedlicher Akteure zu erfassen, wurden 76 halbstrukturierte Interviews mit Experten aus der Wissenschaft $(n=16)$, der Politik ( $n=14$ ) und der betrieblichen Praxis (Planer $n=17$; Betriebsräte $n=14$; Mitarbeitende in der Produktion $n=15$ ) durchgeführt. Die Interviews wurden im Rahmen einer Delphi-Studie durchgeführt und dienten als Ausgangspunkt für die Formulierung von Szenarien zur Zukunft der Produktionsarbeit. Für Delphi-Studien wird empfohlen pro betrachteter Expertengruppe zwischen 10 und 18 Personen zu befragen (Okoli und Pawlowski 2004). Aus diesem Grund umfasst diese Studie eine vergleichsweise hohe Anzahl an Interviews für eine qualitative Studie.

Für die Interviews wurde vorab ein Leitfaden erstellt, der alle für die Befragung relevanten Themengebiete und einen vorbereiteten Pool an Fragen zur Exploration der einzelnen Themengebiete beinhaltete. Die Durchführung von halbstrukturierten Interviews ermöglicht es, beispielsweise die Reihenfolge von Fragen anzupassen und auf die Antworten der einzelnen Interviewteilnehmer eingehen zu können (Kallio et al. 2016; Qu und Dumay 2011). Die Identifikation der Experten erfolgte durch Recherchen (z.B. Veröffentlichungen zum Thema Industrie 4.0, Mitwirkung in relevanten Fachgruppen oder Gremien). Eine Übersicht über die Merkmale und Identifikationskriterien für die Expertengruppen ist in Tab. 1 dargestellt.

Als demografische Variablen wurden das Alter und die Berufserfahrung in Jahren erhoben. Um die Anonymität der Befragten sicherzustellen, wurden diese Variablen kategorial erhoben. Die Altersverteilung der Interviewteilnehmer ist getrennt für die einzelnen Expertengruppen in Abb. 2 und die Berufserfahrung in Abb. 3 dargestellt. Die Altersverteilung der Stichprobe zeigt, dass befragte Experten aus der Gruppe der Anwender und Planer tendenziell jünger waren als befragte Experten aus der Wissenschaft, politische Ak-
Abb. 2 Altersverteilung der Interviewteilnehmer getrennt für die fünf Expertengruppen. (*Ein Interviewteilnehmer machte keine Angaben zum Alter)

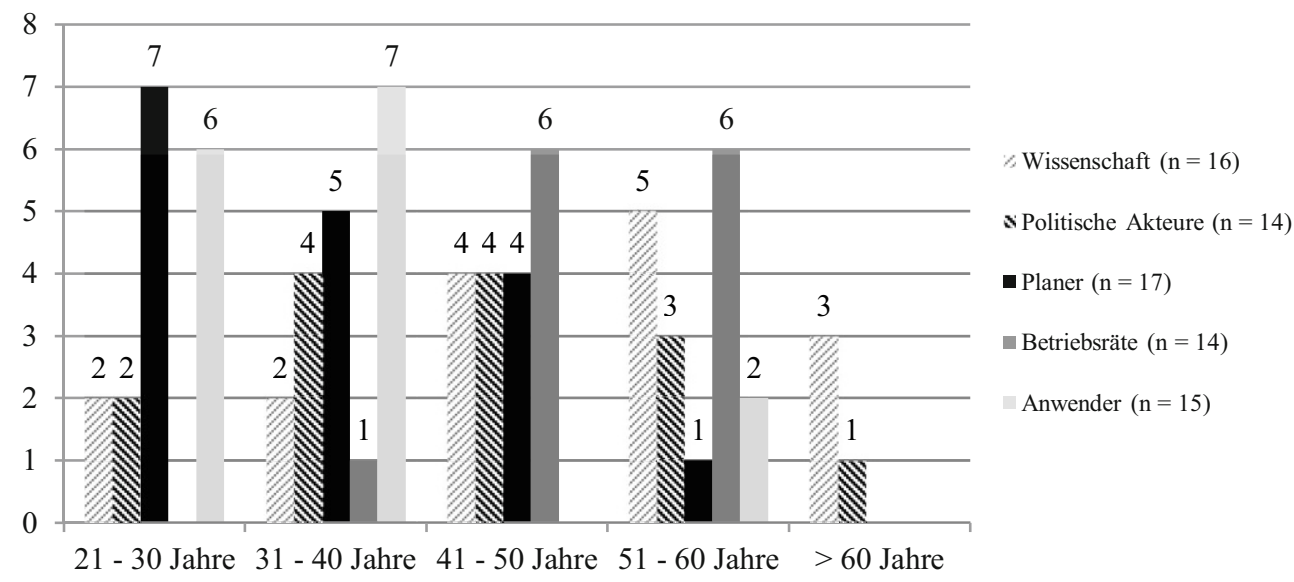


Abb. 3 Berufserfahrung der Interviewteilnehmer getrennt für die fünf Expertengruppen. (*Ein Interviewteilnehmer machte keine Angaben zur Berufserfahrung)

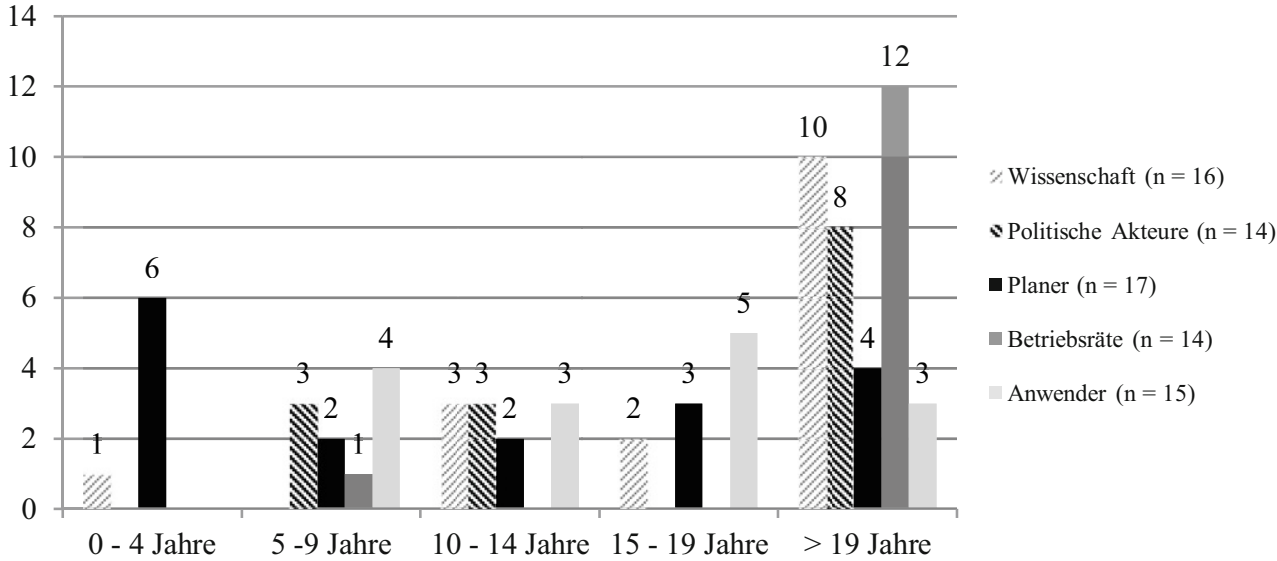

teure und Betriebsräte. Eine ähnliche Verteilung zeigt sich für die Berufserfahrung der Stichprobe. Befragte Experten aus den Gruppen Betriebsrat, Wissenschaft und politische Akteure weisen überwiegend eine langjährige Berufserfahrung (>19 Jahre) auf.

\subsection{Datenaufbereitung und Vorgehen bei der Erstellung des Kategoriensystems}

Die 76 Interviews wurden in MAXQDA (VERBI Software 2017) nach den inhaltich-semantischen Transkriptionsregeln von Dresing und Pehl (2018) transkribiert. AnschlieBend erfolgte die Auswertung der Interviews mittels qualitativer strukturierender Inhaltsanalyse (Steigleder 2008).

Als Grundlage für die Erstellung des Kategoriensystems zu Anwendungsmöglichkeiten von DWAS wurden die Anwendungskategorien aus der Studie von Niemöller et al. (2017) genutzt. Aussagen, die keiner bestehenden Kategorie entsprachen, wurden der Kategorie „Sonstiges“ zugeordnet. Im Anschluss an den ersten Kodiervorgang wurden die Aussagen der Kategorie „Sonstiges“ gesichtet und neue Hauptkategorien entwickelt. Nicht benötigte Hauptkategorien wurden entfernt. Anschließend wurden alle Hauptkategorien detailliert und Subkategorien erstellt. Für die Definition der Subkategorien wurde in Teilen auf die Beschreibungen der Funktionsgruppen von Niemöller et al. (2017) zurückgegriffen. Zusätzlich wurden eigene Subkategorien ergänzt. In einem zweiten Kodierschritt wurden die zuvor identifizierten Textstellen einer Subkategorie zugeordnet.

Als Grundlage für die Entwicklung des Kategoriensystems zu Chancen und Risiken beim Einsatz von DWAS wurden die Dimension Arbeitsgestaltung sowie die sechs Dimensionen zur Technikbewertung aus der VDI-Richtlinie 3780 als Hauptkategorien genutzt (VDI - Verein Deutscher Ingenieure 2000): technische Dimension, wirtschaftliche Dimension, gesundheitliche Dimension, ökologische Dimension, humane Dimension und soziale Dimension. Aussagen, die keiner Hauptkategorie zugeordnet werden konnten, wurden der Kategorie „Sonstiges“ zugeordnet. Anschließend wurden die vorhandenen Hauptkategorien detailliert und Subkategorien erstellt. Zur Erstellung der Subkategorien für die Hauptkategorie Arbeitsgestaltung wurde beispielsweise auf die Unterscheidung in Tätigkeitsund Wissensmerkmale aus dem Work Design Questionnaire (Morgeson und Humphrey 2006; Stegmann et al. 2010) zurückgegriffen. Für die Erstellung von Subkategorien für die Hauptkategorie technologische Dimension wurden die Kriterien Systemqualität (Wahrnehmung des technologischen Systems und der Informationsbereitstellung) und Informationsqualität (Wahrnehmung der Qualität der bereitgestellten Informationen) genutzt, die häufig zur Bewertung von Technologien genutzt werden (Brunkow und Hub 2018; Wixom und Todd 2005).

\section{Ergebnisse}

In diesem Abschnitt werden die Ergebnisse aus den Experteninterviews vorgestellt. $\mathrm{Zu}$ Beginn werden die Kategoriensysteme zu Anwendungsmöglichkeiten von DWAS und Chancen und Risiken beim Einsatz von DWAS mit ihren Haupt- und Subkategorien dargestellt und die Gütekriterien berichtet. Danach werden die Ergebnisse der deskriptiven Datenauswertung für die fünf Expertengruppen vorgestellt.

\subsection{Beschreibung des Kategoriensystems Anwendungsmöglichkeiten von DWAS}

Das Kategoriensystem Anwendungsmöglichkeiten fasst die von den Experten beschriebenen Möglichkeiten zur Anwendung von DWAS in der Produktion zusammen. Es besteht aus den Hauptkategorien (1) Arbeitsschutz, (2) Arbeitstätigkeit, (3) Identifizierung, (4) Kommunikation, (5) Management, (6) Navigation, (7) Organisation, (8) Qualifizierung und Kompetenzentwicklung und (9) Qualitätssicherung. Den neun Hauptkategorien sind 45 Subkategorien zu- 
Tab. 2 Übersicht Kategoriensystem Anwendungsmöglichkeiten von DWAS in Fertigung, Montage und Logistik

\begin{tabular}{|c|c|c|c|}
\hline & Arbeitsschutz & Arbeitstätigkeit & Identifizierung \\
\hline Definition & $\begin{array}{l}\text { DWAS werden eingesetzt, um Mitarbei- } \\
\text { tende vor schädlichen Belastungen und } \\
\text { Gefahren zu schützen }\end{array}$ & $\begin{array}{l}\text { DWAS werden eingesetzt, um Mitarbei- } \\
\text { tende bei der Erfüllung der Arbeitsaufga- } \\
\text { be zu unterstützen }\end{array}$ & $\begin{array}{l}\text { DWAS werden eingesetzt, um Mitar- } \\
\text { beitende und Objekte zu identifizieren }\end{array}$ \\
\hline \multirow[t]{5}{*}{ Subkategorien } & Ergonomie sicherstellen & Feedback zur Ausführung & Anmeldung an Arbeitsplätzen \\
\hline & $\begin{array}{l}\text { Prozessbezogene Warn- und Sicherheits- } \\
\text { hinweise anzeigen }^{\mathrm{a}}\end{array}$ & Schritt-für-Schritt-Anleitung (dauerhaft) & Barcodes und QR-Codes scannen ${ }^{\mathrm{a}}$ \\
\hline & $\begin{array}{l}\text { Objektbezogene Warn- und Sicherheits- } \\
\text { hinweise anzeigen }{ }^{\mathrm{a}}\end{array}$ & Temporäre Unterstützung & Informationen zum Objekt anzeigen $^{\mathrm{a}}$ \\
\hline & Verhaltensprävention & $\begin{array}{l}\text { Weiterführende Informationen zur Ar- } \\
\text { beitstätigkeit }\end{array}$ & $\begin{array}{l}\text { Objekte mit Objekterkennung } \\
\text { identifizieren }^{\mathrm{a}}\end{array}$ \\
\hline & Kommunikation & Management & Navigation \\
\hline Definition & $\begin{array}{l}\text { DWAS werden eingesetzt um innerhalb } \\
\text { von Teams und über die Teamgrenzen } \\
\text { hinweg zu kommunizieren }\end{array}$ & $\begin{array}{l}\text { DWAS werden eingesetzt, um Prozesse } \\
\text { zu erfassen, abzubilden und zu steuern }\end{array}$ & $\begin{array}{l}\text { DWAS werden für die Navigation } \\
\text { eingesetzt }\end{array}$ \\
\hline \multirow[t]{11}{*}{ Subkategorie } & $\begin{array}{l}\text { Arbeitsschritte per Fernsteuerung anlei- } \\
\text { ten (Support) }\end{array}$ & Belohnungssymbole einblenden ${ }^{a}$ & $\begin{array}{l}\text { Echtzeitkarten mit Verkehrsinfos } \\
\text { darstellen (dynamisch) }\end{array}$ \\
\hline & $\begin{array}{l}\text { Erreichbarkeit von Vorgesetzten, Team- } \\
\text { mitgliedern oder anderen Bereichen }\end{array}$ & Decision-Support-System & $\begin{array}{l}\text { Navigationsanweisungen erteilen } \\
\text { (statisch) }^{\mathrm{a}}\end{array}$ \\
\hline & \multirow{8}{*}{$\begin{array}{l}\text { Videokommunikation zur gemeinsamen } \\
\text { Problemlösung }\end{array}$} & Mitarbeiter Arbeitsprozessen zuordnen & \\
\hline & & $\begin{array}{l}\text { Mitarbeiter auf Basis von gesundheitli- } \\
\text { chen Merkmalen priorisieren }\end{array}$ & \\
\hline & & Monitoring - Prozesse erfassen ${ }^{\mathrm{a}}$ & \\
\hline & & Monitoring - Auswertungen anzeigen ${ }^{\mathrm{a}}$ & \\
\hline & & Monitoring - Simulation & \\
\hline & & Performance Tracking & \\
\hline & & $\begin{array}{l}\text { Personenbezogene Austaktung der } \\
\text { Arbeitsaufgabe }\end{array}$ & \\
\hline & & Standort erfassen & \\
\hline & Organisation & $\begin{array}{l}\text { Qualifizierung und Kompetenzent- } \\
\text { wicklung }\end{array}$ & Qualitätssicherung \\
\hline Definition & $\begin{array}{l}\text { DWAS werden eingesetzt, um Abläufe } \\
\text { und koordinative Tätigkeiten zu unter- } \\
\text { stützen }\end{array}$ & $\begin{array}{l}\text { DWAS werden eingesetzt, um Lern- und } \\
\text { Anlernprozesse zu unterstützen }\end{array}$ & $\begin{array}{l}\text { DWAS werden eingesetzt, um die } \\
\text { Qualität von Produkten und Abläufen } \\
\text { sicherzustellen }\end{array}$ \\
\hline \multirow[t]{7}{*}{ Subkategorie } & Automatisierte Bestellvorgänge & Anlernen & $\begin{array}{l}\text { Automatisierte Kontrolle des Objekt- } \\
\text { zustands durchführen }^{\text {a }}\end{array}$ \\
\hline & Ideenmanagement & Digitales Lernen im Prozess der Arbeit & $\begin{array}{l}\text { Automatisierte Kontrollfunktion } \\
\text { unterstützen }^{\mathrm{a}}\end{array}$ \\
\hline & Inklusion & $\begin{array}{l}\text { Kompetenzniveau-angepasste Unterstüt- } \\
\text { zung }\end{array}$ & Fehler dokumentieren ${ }^{\mathrm{a}}$ \\
\hline & Planung der An- und Abwesenheiten & Selbstgesteuertes Lernen im Medien & Prozessausführung dokumentieren ${ }^{\mathrm{a}}$ \\
\hline & $\begin{array}{l}\text { Qualifikation dokumentieren und ver- } \\
\text { walten }\end{array}$ & Wissensmanagement & Prüfprozesse unterstützen \\
\hline & Schichtübergabe & & \\
\hline & Zusatzinformationen & & \\
\hline
\end{tabular}

${ }^{a}$ Kategorien sind aus der Studie von Niemöller et al. (2017) entnommen

geordnet. Das Kategoriensystem ist in Tab. 2 dargestellt. Die Kategorie Arbeitsschutz beschreibt die Anwendung von DWAS zum Schutz der Mitarbeitenden vor schädlichen Belastungen oder Gefahren. Die Hauptkategorie Arbeitstätigkeit beinhaltet Aussagen dazu, dass DWAS die Mitarbeitenden durch die Bereitstellung von Informationen bei der Erfüllung der Arbeitsaufgabe unterstützen. In der Hauptkategorie Identifizierung werden Aussagen der
Interviewteilnehmer zusammengefasst, die beschreiben, dass DWAS eingesetzt werden, um Mitarbeitende oder Objekte zu identifizieren. Die Hauptkategorie Kommunikation beschreibt die Nutzung von DWAS, um innerhalb von Teams oder über Teamgrenzen hinweg zu kommunizieren. Die Hauptkategorie Management beschreibt, dass DWAS genutzt werden, um Prozesse und Abläufe sichtbar zu machen, zu überwachen und zu steuern (Niemöller 
et al. 2017). Die Hauptkategorie Navigation beschreibt die Anwendung von DWAS zum Erteilen statischer oder dynamischer Navigationsanweisungen (Niemöller et al. 2017). In der Hauptkategorie Organisation sind Anwendungsfälle zusammengefasst, in denen DWAS für die Koordination und Organisation von Abläufen eingesetzt werden. Die Hauptkategorie Qualifizierung und Kompetenzentwicklung beschreibt den Einsatz von DWAS zur Qualifizierung und zum Lernen. In der Hauptkategorie Qualitätssicherung werden Anwendungsmöglichkeiten von DWAS zur Sicherstellung und Steigerung der Produktqualität erfasst.

\subsection{Beschreibung des Kategoriensystems Chancen und Risiken beim Einsatz von DWAS}

Das Kategoriensystem zu Chancen und Risiken beim Einsatz von DWAS in der Produktion besteht aus den Hauptkategorien (1) Arbeitsgestaltung, (2) Individuelle Dimension, (3) Ökologie, (4) Ökonomie, (5) Soziale Dimension und (6) Technologische Dimension. Den sechs Hauptkategorien sind 26 Subkategorien zugeordnet. Die Kategorien im Kategoriensystem sind bewusst sprachlich neutral gehalten, um sowohl Chancen als auch Risiken mit dem gleichen Kategoriensystem erfassen zu können. Das Kategoriensystem ist in Tab. 3 dargestellt.

In der Hauptkategorie Arbeitsgestaltung sind Chancen und Risiken von DWAS für die Gestaltung der Arbeitstätigkeit und der Arbeitsorganisation zusammengefasst. Die Hauptkategorie individuelle Dimension enthält Aussagen der Interviewteilnehmer zu Chancen und Risiken, die sich für den individuellen Mitarbeitenden ergeben. Die Hauptkategorie Ökologie beinhaltet Aussagen der Interviewteilnehmer zu Chancen und Risiken im Umgang mit Ressourcen. In der Hauptkategorie Ökonomie sind wirtschaftliche Chancen und Risiken beim Einsatz von DWAS zusammengefasst. Die Hauptkategorie soziale Dimension erfasst Chancen und Risiken für die Beziehung und den Austausch zwischen Mitarbeitenden. Die Hauptkategorie technologische Dimension beschreibt Chancen und Risiken, die sich für die Informationsdarbietung und die Qualität der Informationen ergeben.

\subsection{Gütekriterien}

Nach einer ersten Gesamtkodierung des Materials wurde die Intercoder-Reliabilität (Kappa, $\kappa_{\mathrm{n}}$ Brennan und Prediger 1981) bestimmt, um die Qualität des Kodierprozesses zu ermitteln und zu verbessern (Rädiker und Kuckartz 2019). Hierfür wurde für zwei Personen, die im Rahmen einer hilfswissenschaftlichen Tätigkeit das Forschungsprojekt unterstützten, eine Kodierschulung durchgeführt. Danach fand mit zwei zufällig ausgewählten Interviews ein erster Kodiervorgang statt. Die Ergebnisse wurden anschließend ausgewertet und diskutiert. Nicht-Übereinstimmungen bildeten die Grundlage für die Überarbeitung und Schärfung der Kategoriensysteme und der Kodierleitfäden. Das gesamte Datenmaterial wurde mit den angepassten Kategoriensystemen erneut kodiert. Danach wurden für jeden der beiden zuvor geschulten Kodierer elf Interviews (ca. 15\% des Gesamtmaterials) zufällig ausgewählt und mit einem der beiden Kategoriensysteme zweitkodiert.

Für die kodierten Textstellen mit dem Kategoriensystem Anwendungsmöglichkeiten lag Kappa bei $\kappa_{\mathrm{n}}=0,88$. Die Kappa-Werte der einzelnen Interviews lagen zwischen $\kappa_{\mathrm{n}}=0,81$ und $\kappa_{\mathrm{n}}=0,96$. Für das Kategoriensystem Chancen und Risiken wurde insgesamt ein Kappa von $\kappa_{\mathrm{n}}=0,84$ erreicht. Die Kappa-Werte der einzelnen Interviews lagen zwischen $\kappa_{n}=0,72$ und $\kappa_{n}=1,00$. Bei der Interpretation der erreichten Kappa-Werte kann auf die etablierten Konventionen zur Interpretation von Cohens Kappa zurückgegriffen werden (Rädiker und Kuckartz 2019). Ab einem Kappa von $\kappa>0,80$ kann von einer sehr guten Übereinstimmung ausgegangen werden.

\subsection{Deskriptive Datenauswertung und Gruppenvergleiche}

Nachfolgend werden die Ergebnisse der deskriptiven Datenauswertung für die beiden Themen Anwendungsmöglichkeiten und Chancen und Risiken beim Einsatz von DWAS vorgestellt. Weiterhin werden die Antworten der unterschiedlichen Expertengruppen miteinander verglichen.

\subsubsection{Deskriptive Ergebnisse und Gruppenvergleiche zu Anwendungsmöglichkeiten von DWAS}

In Tab. 4 sind die absoluten und prozentualen Nennhäufigkeiten für Anwendungsmöglichkeiten von DWAS für jede der fünf Expertengruppen dargestellt. Über alle Expertengruppen hinweg werden die Anwendungsmöglichkeiten Arbeitstätigkeit, Qualitätssicherung und Management am häufigsten genannt. Eine Besonderheit ergibt sich bei den Expertengruppen Wissenschaft und politische Akteure. Hier wird zusätzlich die Anwendungsmöglichkeit Qualifizierung und Kompetenzentwicklung sehr häufig genannt. Die geringe Nennhäufigkeit der Anwendungsmöglichkeit Navigation über alle Expertengruppen hinweg verwundert, da in die Fragestellung der Studie explizit die Anwendung von DWAS in den Gewerken Fertigung, Montage und Logistik adressiert wurde und der Transport von Gütern und Waren einen relevanten Anteil an der Arbeitstätigkeit ausmacht.

In Abb. 4 sind die prozentualen Nennhäufigkeiten grafisch für jede Gruppe dargestellt. Durch diese Darstellung lassen sich besonders gut Gemeinsamkeiten und Unterschiede hinsichtlich der Nennhäufigkeiten für die unterschiedlichen Expertengruppen betrachten. Die An- 
Tab. 3 Übersicht Kategoriensystem Chancen und Risiken beim Einsatz von DWAS in Fertigung, Montage und Logistik

\begin{tabular}{llll}
\hline Arbeitsgestaltung & Individuelle Dimension & Ökologie \\
\hline Definition & Chancen und Risiken beim & Chancen und Risiken beim Einsatz von & Chancen und Risiken beim Einsatz von DWAS für \\
& $\begin{array}{c}\text { Einsatz von DWAS für die } \\
\text { Gestaltung der Arbeitstätig- } \\
\text { keit, des Arbeitsplatzes und }\end{array}$ & DWAS für den individuellen Mitarbeiten- & die Umwelt \\
& &
\end{tabular}
der Arbeitsorganisation

Zitat: „Es entsteht jetzt die Möglichkeit auch komplexere Tätigkeiten, also Job Enrichment, Job Enlargement, zu machen, weil einfach die Unterstützungsmöglichkeiten da sind." (IT27, Wissenschaft)

\section{Subkate- Arbeitsintensität (z. B. Ar- gorien beitsverdichtung vs. indivi- duelle Austaktung)}

Arbeitszeit (z. B. Erreichbarkeit vs. Flexibilisierung)

Entlohnung

Ergonomie

Monitoring (z. B. Kontrolle der Mitarbeitenden)

Tätigkeitsmerkmale (Morgeson und Humphrey 2006; Stegmann et al. 2010) (z. B. Aufgabenvielfalt, Autonomie)

Wissensmerkmale (Morgeson und Humphrey 2006; Stegmann et al. 2010) (z. B. Anforderungsvielfalt)

\begin{tabular}{|c|c|c|c|}
\hline & Ökonomie & Soziale Dimension & Technologische Dimension \\
\hline \multirow[t]{2}{*}{ Definition } & $\begin{array}{l}\text { Chancen und Risiken beim } \\
\text { Einsatz von DWAS für die } \\
\text { Wirtschaftlichkeit des Un- } \\
\text { ternehmens }\end{array}$ & $\begin{array}{l}\text { Chancen und Risiken beim Einsatz von } \\
\text { DWAS für die Beziehungen und den } \\
\text { Austausch der Mitarbeitenden }\end{array}$ & $\begin{array}{l}\text { Chancen und Risiken beim Einsatz von DWAS für } \\
\text { die Technologienutzung und -gestaltung }\end{array}$ \\
\hline & $\begin{array}{l}\text { Zitat: „Wenn ich natürlich } \\
\text { künftig solche Wearables } \\
\text { habe, die mir Schritt für } \\
\text { Schritt vorgeben, könn- } \\
\text { te man das natürlich als } \\
\text { Chance nehmen, um zu sa- } \\
\text { gen naja, dadurch werden } \\
\text { auch Fehlerwahrschein- } \\
\text { lichkeiten reduziert.“ (IT5, } \\
\text { Betriebsrat) }\end{array}$ & $\begin{array}{l}\text { Zitat: „Also die Chancen bestehen ganz } \\
\text { klar darin [...] neue Möglichkeiten der } \\
\text { internen Vernetzung [zu] bieten, der in- } \\
\text { ternen Zusammenarbeit.“ (IT25, Wissen- } \\
\text { schaft) }\end{array}$ & $\begin{array}{l}\text { Zitat: Und die andere Gefahrendiskussion ist schon } \\
\text { die, dass diese neuen Technologien so eingesetzt } \\
\text { werden, dass sie eher arbeitserschwerend [sind], } \\
\text { Belastungen erzeugen, Informationsüberflutung, Un- } \\
\text { sicherheiten, nicht genügend Qualifizierung. [...] } \\
\text { Also immer dann, wenn Technologien nicht gut } \\
\text { funktionieren, geht das einerseits auf Kosten des } \\
\text { Unternehmens [...]. Auf der anderen Seite geht es } \\
\text { aber eigentlich fast immer auf Kosten der Leute. Weil } \\
\text { die müssten dann diese Dinge kompensieren. (IT27, } \\
\text { Wissenschaft) }\end{array}$ \\
\hline \multirow[t]{5}{*}{$\begin{array}{l}\text { Subkate- } \\
\text { gorie }\end{array}$} & Effizienz & Inklusion/Exklusion & $\begin{array}{l}\text { Informationsqualität (Brunkow und Hub 2018; Wi- } \\
\text { xom und Todd 2005) (z. B. Datenverfügbarkeit, Dar- } \\
\text { stellungsmöglichkeiten) }\end{array}$ \\
\hline & Kosten & Kommunikation & Systemqualität (Brunkow und Hub 2018; Wixom und \\
\hline & $\begin{array}{l}\text { Personal (z. B. Personalab- } \\
\text { bau) }\end{array}$ & Partizipation & $\begin{array}{l}\text { Todd 2005) (z. B. Benutzerfreundlichkeit, Vertrauen, } \\
\text { Datenschutz) }\end{array}$ \\
\hline & Produktivität & Zusammenarbeit & \\
\hline & Qualität & & \\
\hline
\end{tabular}

Zitat: „Diese Verschwendung an Rohstoffen würde wegfallen, indem man einfach nur ein Tablet am Stapler installiert, wo die Informationen direkt einfließen." (IT49, Betriebsrat)

Umgang mit Ressourcen
Gesundheit (z. B. physische und psychische Folgen)

Motivation/Demotivation

Selbstwert

Technikglaube (z. B. Misstrauen, Datengläubigkeit)

Work-Life-Balance (z. B. individuelle Flexibilisierung) Technologien auch auf die Psyche. Also, Konzentration, Dinge schnell einschätbelastbar ist unser Brain?" (IT48, politischer Akteur)

Employability
Zitat: „Aber die Gefahren wie gesagt, [...]

-


Tab. 4 Übersicht der absoluten und prozentualen Häufigkeiten der Antworten für jede einzelne Expertengruppe zum Thema Anwendungsmöglichkeiten

\begin{tabular}{|c|c|c|c|c|c|c|c|c|c|c|}
\hline \multirow{3}{*}{$\begin{array}{l}\text { Kategorie } \\
\text { Arbeitsschutz }\end{array}$} & \multirow{2}{*}{\multicolumn{2}{|c|}{$\begin{array}{l}\text { Wissenschaftler } \\
(n=16) \\
\text { Häufigkeiten\% }\end{array}$}} & \multirow{2}{*}{\multicolumn{2}{|c|}{$\begin{array}{l}\begin{array}{l}\text { Politische Akteure } \\
(\mathrm{n}=14)\end{array} \\
\text { Häufigkeiten } \%\end{array}$}} & \multirow{2}{*}{\multicolumn{2}{|c|}{$\begin{array}{l}\begin{array}{l}\text { Planer } \\
(n=17)\end{array} \\
\text { Häufigkeiten } \%\end{array}$}} & \multirow{2}{*}{\multicolumn{2}{|c|}{$\begin{array}{l}\begin{array}{l}\text { Betriebsräte } \\
(\mathrm{n}=14)\end{array} \\
\text { Häufigkeiten } \%\end{array}$}} & \multirow{2}{*}{\multicolumn{2}{|c|}{$\begin{array}{l}\begin{array}{l}\text { Anwender } \\
(n=15)\end{array} \\
\text { Häufigkeiten\% }\end{array}$}} \\
\hline & & & & & & & & & & \\
\hline & 7 & 44 & 8 & 57 & 3 & 18 & 6 & 43 & 1 & 7 \\
\hline Arbeitsstätigkeit & 15 & 94 & 14 & 100 & 16 & 94 & 9 & 64 & 12 & 80 \\
\hline Identifizierung & 6 & 38 & 6 & 43 & 10 & 59 & 6 & 43 & 7 & 47 \\
\hline Kommunikation & 11 & 69 & 9 & 64 & 11 & 65 & 6 & 43 & 8 & 53 \\
\hline Management & 15 & 94 & 13 & 93 & 13 & 77 & 12 & 86 & 9 & 60 \\
\hline Navigation & 3 & 19 & 2 & 14 & 7 & 35 & 1 & 7 & 3 & 20 \\
\hline Organisation & 6 & 38 & 7 & 50 & 8 & 41 & 7 & 50 & 5 & 53 \\
\hline $\begin{array}{l}\text { Qualifizierung und } \\
\text { Kompetenzentwick- } \\
\text { lung }\end{array}$ & 15 & 94 & 10 & 71 & 9 & 47 & 4 & 29 & 1 & 7 \\
\hline Qualitätssicherung & 12 & 75 & 10 & 71 & 14 & 82 & 10 & 71 & 10 & 67 \\
\hline
\end{tabular}

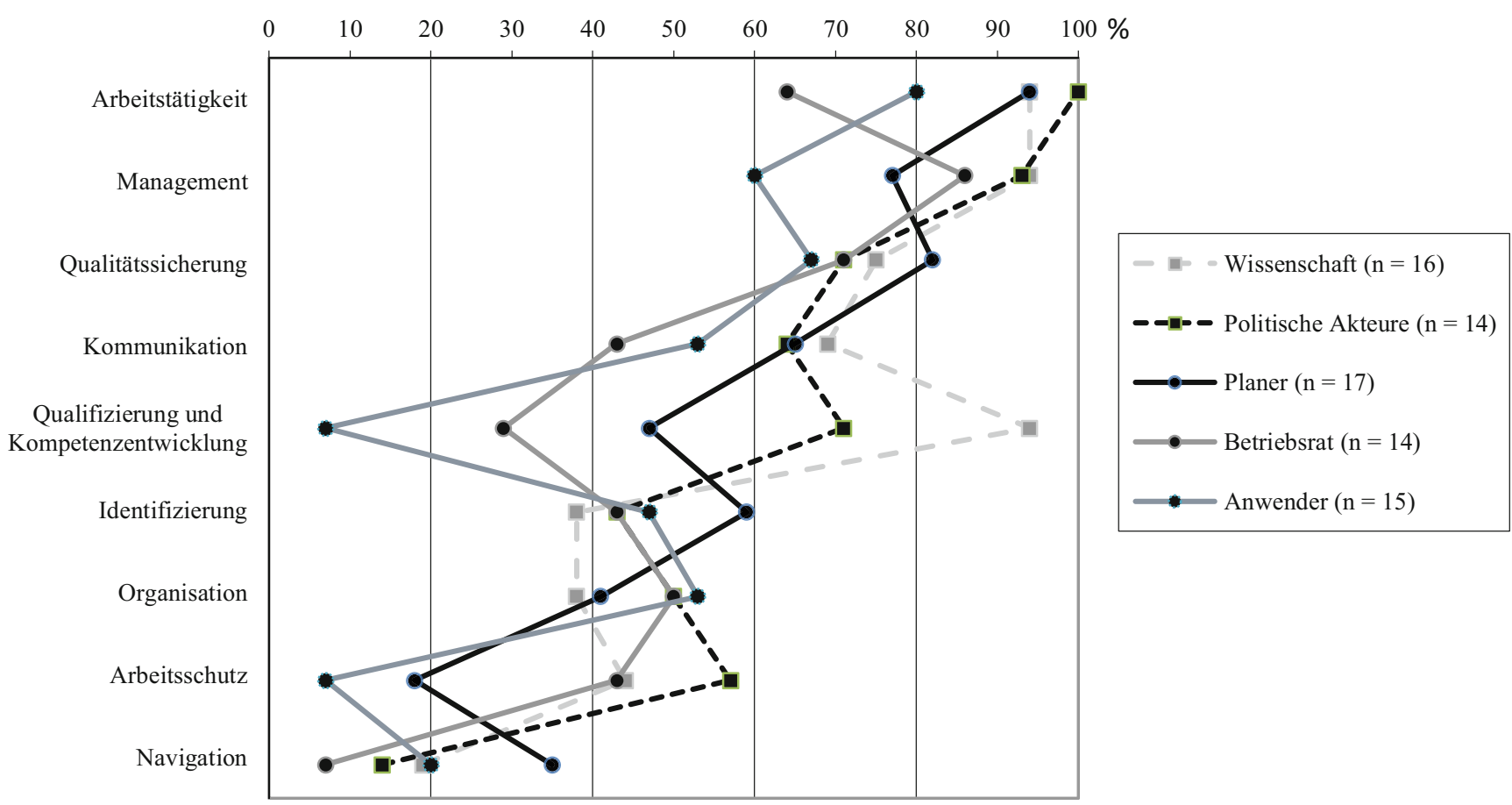

Abb. 4 Zusammenfassende Übersicht der prozentualen Häufigkeiten der Antworten aller Expertengruppen zum Thema Anwendungsmöglichkeiten

wendungsmöglichkeiten Qualitätssicherung, Organisation und Identifizierung werden über die Expertengruppen hinweg ähnlich häufig genannt. Große Unterschiede zeigen sich vor allem für das Anwendungsfeld Qualifizierung und Kompetenzentwicklung. Hier liegen die Nennhäufigkeiten der einzelnen Expertengruppen sehr weit auseinander. Am häufigsten wird diese Anwendungsmöglichkeit von Wissenschaftlern genannt (in $94 \%$ der Interviews), am seltensten von Anwendern (in 7\% der Interviews).

\subsubsection{Deskriptive Ergebnisse und Gruppenvergleiche zu Chancen und Risiken beim Einsatz von DWAS}

In Summe wurden 940 Textstellen identifiziert, in denen Chancen oder Risiken beim Einsatz von DWAS in der Produktion benannt wurden. 448 Codes entfielen auf benannte Chancen und 492 Codes auf benannte Risiken. Tab. 5 und 6 zeigen die absoluten und prozentualen Häufigkeiten der benannten Chancen und Risiken beim Einsatz von DWAS je Expertengruppe.

Abb. 5 zeigt grafisch, wie sich die Nennungen von Chancen und Risiken je Expertengruppe auf die sechs Hauptka- 
Tab. 5 Übersicht der absoluten und prozentualen Häufigkeiten der Antworten für jede einzelne Expertengruppe zum Thema Chancen beim Einsatz von DWAS

\begin{tabular}{|c|c|c|c|c|c|c|c|c|c|c|}
\hline \multirow[t]{2}{*}{ Kategorie } & \multicolumn{2}{|c|}{$\begin{array}{l}\text { Wissenschaftler } \\
(n=16)\end{array}$} & \multicolumn{2}{|c|}{$\begin{array}{l}\text { Politische Akteure } \\
(n=14)\end{array}$} & \multicolumn{2}{|l|}{$\begin{array}{l}\text { Planer } \\
(n=17)\end{array}$} & \multicolumn{2}{|l|}{$\begin{array}{l}\text { Betriebsräte } \\
(\mathrm{n}=14)\end{array}$} & \multicolumn{2}{|l|}{$\begin{array}{l}\text { Anwender } \\
(n=15)\end{array}$} \\
\hline & Häufigkeiten & $\%$ & Häufigkeiten & $\%$ & Häufigkeiten & $\%$ & Häufigkeiten & $\%$ & Häufigkeiten & $\%$ \\
\hline Arbeitsgestaltung & 14 & 88 & 11 & 79 & 8 & 47 & 8 & 57 & 3 & 20 \\
\hline Arbeitsintensität & 1 & 6 & 1 & 7 & - & - & - & - & - & - \\
\hline Arbeitszeit & 1 & 6 & 6 & 43 & - & - & 1 & 7 & - & - \\
\hline Entlohnung & - & - & - & - & - & - & - & - & - & - \\
\hline Ergonomie & 3 & 19 & 3 & 21 & - & - & 4 & 29 & - & - \\
\hline Monitoring & - & - & - & - & 1 & 6 & - & - & - & - \\
\hline Tätigkeitsmerkmale & 8 & 50 & 8 & 57 & 3 & 18 & 1 & 7 & - & - \\
\hline Wissensmerkmale & 13 & 81 & 7 & 50 & 7 & 41 & 2 & 14 & 3 & 20 \\
\hline Individuelle Dimension & 6 & 38 & 7 & 50 & 9 & 53 & 2 & 14 & 1 & 7 \\
\hline Employability & - & - & - & - & 2 & 12 & - & - & - & - \\
\hline Gesundheit & 3 & 19 & 4 & 29 & 2 & 12 & 1 & 7 & 1 & 7 \\
\hline Motivation & 3 & 19 & 1 & 7 & 4 & 24 & 1 & 7 & - & - \\
\hline Selbstwert & 1 & 6 & 2 & 14 & 4 & 24 & - & - & - & - \\
\hline Technikumgang & - & - & - & - & - & - & - & - & - & - \\
\hline Work-Life-Balance & - & - & - & - & - & - & - & - & - & - \\
\hline Ökologische Dimension & 1 & 6 & - & - & 2 & 12 & 1 & 7 & 2 & 13 \\
\hline Ökonomische Dimension & 11 & 69 & 13 & 93 & 13 & 77 & 11 & 79 & 10 & 67 \\
\hline Effizienz & 7 & 44 & 7 & 50 & 10 & 59 & 8 & 57 & 7 & 47 \\
\hline Kosten & - & - & - & - & 2 & 12 & - & - & - & - \\
\hline Personal & 1 & 6 & - & - & 2 & 12 & - & - & 1 & 7 \\
\hline Produktivität & 1 & 6 & 7 & 50 & 4 & 24 & 2 & 14 & 1 & 7 \\
\hline Qualität & 6 & 38 & 7 & 50 & 6 & 35 & 8 & 57 & 7 & 33 \\
\hline Wettbewerbsfähigkeit & 3 & 19 & 4 & 29 & 3 & 18 & 1 & 7 & - & - \\
\hline Soziale Dimension & 5 & 31 & 7 & 50 & 3 & 18 & 4 & 14 & 4 & 27 \\
\hline Inklusion & 1 & 6 & 3 & 21 & - & - & - & - & 1 & 7 \\
\hline Kommunikation & 2 & 13 & 2 & 14 & 3 & 18 & 1 & 7 & 2 & 13 \\
\hline Partizipationsmöglichkeiten & 1 & 6 & - & - & - & - & - & - & 1 & 7 \\
\hline Zusammenarbeit & 4 & 25 & 3 & 21 & 1 & 6 & 1 & 7 & - & - \\
\hline Technologische Dimension & 12 & 75 & 11 & 79 & 13 & 77 & 8 & 57 & 5 & 33 \\
\hline Informationsqualität & 11 & 69 & 11 & 79 & 12 & 71 & 7 & 50 & 5 & 33 \\
\hline Systemqualität & 2 & 13 & 1 & 7 & 3 & 18 & 2 & 14 & 2 & 13 \\
\hline
\end{tabular}

Pro Interview wird nur eine Nennung der jeweiligen Subkatgorie/Kategorie erfasst

tegorien verteilen. Chancen werden vor allem für die Dimensionen Ökonomie und Technologie benannt. Chancen für die Ökonomie werden vor allem in einer gesteigerten Effizienz und einer verbesserten Qualität (z.B. durch eine geringere Fehlerquote) gesehen. Chancen für die technologische Dimension werden vor allem von den Expertengruppen politische Akteure, Planer und Wissenschaftler genannt. Obwohl von den Gruppen Betriebsrat und Anwender seltener Chancen für die technologische Dimension benannt werden, stimmen die Gruppennennungen darin überein, dass vor allem Verbesserung der Informationsqualität vorgebracht werden. Dabei werden vor allem eine höhere Prozesstransparenz durch das Vorhandensein und die Nutzung der verfügbaren Daten und Informationen sowie die
Möglichkeit zur Individualisierbarkeit der Informationsdarstellung als Chance beschrieben.

Risiken werden vor allem für die Dimension Arbeitsgestaltung und die individuelle Dimension des Mitarbeitenden benannt. Die Gruppen Wissenschaft, politische Akteure, Planer und Betriebsrat benennen über alle Kategorien hinweg am häufigsten Risiken für die Arbeitsgestaltung. Dabei werden vor allem Risiken für die Subkategorien Wissensmarkmale, Monitoring und Tätigkeitsmerkmale beschrieben. Bei Wissensmerkmalen benennen die Experten die Sorge davor, dass Mitarbeitende durch den Einsatz von DWAS zukünftig weniger Wissen und Fähigkeiten benötigen, um ihre Arbeit zu bewältigen und dadurch langfristig eine Dequalifizierung stattfindet. Bei der Subkategorie Monitoring beschreiben die Interviewteilnehmer, 
Tab. 6 Übersicht der absoluten und prozentualen Häufigkeiten der Antworten für jede einzelne Expertengruppe zum Thema Risiken beim Einsatz von DWAS

\begin{tabular}{|c|c|c|c|c|c|c|c|c|c|c|}
\hline \multirow[t]{2}{*}{ Kategorie } & \multicolumn{2}{|c|}{$\begin{array}{l}\text { Wissenschaftler } \\
(n=16)\end{array}$} & \multicolumn{2}{|c|}{$\begin{array}{l}\text { Politische Akteure } \\
(\mathrm{n}=14)\end{array}$} & \multicolumn{2}{|l|}{$\begin{array}{l}\text { Planer } \\
(n=17)\end{array}$} & \multicolumn{2}{|l|}{$\begin{array}{l}\text { Betriebsräte } \\
(\mathrm{n}=14)\end{array}$} & \multicolumn{2}{|l|}{$\begin{array}{l}\text { Anwender } \\
(n=15)\end{array}$} \\
\hline & Häufigkeiten & $\%$ & Häufigkeiten & $\%$ & Häufigkeiten & $\%$ & Häufigkeiten & $\%$ & Häufigkeiten & $\%$ \\
\hline Arbeitsgestaltung & 13 & 81 & 13 & 93 & 11 & 65 & 13 & 93 & 5 & 33 \\
\hline Arbeitsintensität & 2 & 13 & 8 & 57 & - & - & 5 & 36 & - & - \\
\hline Arbeitszeit & 1 & 6 & - & - & - & - & - & - & - & - \\
\hline Entlohnung & - & - & 1 & 7 & - & - & 3 & 21 & 1 & 7 \\
\hline Ergonomie & 1 & 6 & - & - & - & - & 1 & 7 & 1 & 7 \\
\hline Monitoring & 8 & 50 & 4 & 29 & 5 & 29 & 7 & 50 & 2 & 13 \\
\hline Tätigkeitsmerkmale & 6 & 38 & 5 & 36 & 6 & 35 & 7 & 50 & 2 & 13 \\
\hline Wissensmerkmale & 10 & 63 & 10 & 71 & 7 & 41 & 8 & 57 & 3 & 20 \\
\hline Individuelle Dimension & 10 & 63 & 12 & 86 & 10 & 59 & 9 & 64 & 6 & 40 \\
\hline Employability & 2 & 13 & 4 & 29 & - & - & - & - & - & - \\
\hline Gesundheit & 6 & 38 & 8 & 57 & 4 & 24 & 5 & 36 & 4 & 27 \\
\hline Motivation & 4 & 25 & - & - & 1 & 6 & 1 & 7 & - & - \\
\hline Selbstwert & 4 & 25 & 4 & 29 & 4 & 24 & 1 & 7 & 1 & 7 \\
\hline Technikumgang & 3 & 19 & 3 & 21 & 4 & 24 & 4 & 29 & 1 & 7 \\
\hline Work-Life-Balance & 1 & 6 & 2 & 14 & 2 & 12 & 1 & 7 & - & - \\
\hline Ökologische Dimension & - & - & - & - & - & - & - & - & - & - \\
\hline Ökonomische Dimension & 3 & 19 & 5 & 36 & 4 & 24 & 5 & 36 & 4 & 27 \\
\hline Effizienz & 1 & 6 & - & - & 1 & 6 & - & - & - & - \\
\hline Kosten & 3 & 19 & 1 & 7 & 2 & 12 & - & - & - & - \\
\hline Personal & 1 & 6 & 4 & 29 & 2 & 12 & 4 & 29 & 4 & 27 \\
\hline Produktivität & - & - & 1 & 7 & 1 & 6 & 1 & 7 & - & - \\
\hline Qualität & - & - & - & - & - & - & - & - & - & - \\
\hline Wettbewerbsfähigkeit & - & - & 3 & 21 & - & - & - & - & - & - \\
\hline Soziale Dimension & 2 & 13 & 6 & 43 & 5 & 29 & 5 & 36 & 4 & 27 \\
\hline Exklusion & 1 & 6 & 1 & 7 & - & - & - & - & - & - \\
\hline Kommunikation & 1 & 6 & 2 & 14 & 3 & 18 & 4 & 29 & 2 & 13 \\
\hline Partizipationsmöglichkeiten & 1 & 6 & 2 & 14 & 1 & 6 & 1 & 7 & - & - \\
\hline Zusammenarbeit & 1 & 6 & 5 & 36 & 3 & 18 & 3 & 21 & 2 & 13 \\
\hline Technologische Dimension & 6 & 38 & 9 & 64 & 10 & 59 & 8 & 57 & 11 & 73 \\
\hline Informationsqualität & 5 & 31 & 5 & 36 & 2 & 12 & 5 & 36 & 2 & 13 \\
\hline Systemqualität & 5 & 31 & 8 & 57 & 9 & 53 & 6 & 43 & 10 & 67 \\
\hline
\end{tabular}

Pro Interview wird nur eine Nennung der jeweiligen Subkatgorie/Kategorie erfasst

dass Mitarbeitende durch DWAS stärker kontrollierbar sind (z.B. Arbeitstempo, Arbeitsleistung) und dadurch zum gläsernen Mitarbeitenden werden. Bei der Subkategorie Tätigkeitsmerkmale benennen die Interviewteilnehmer vor allem die Verschlechterung der Autonomie sowie der Reduzierung der Aufgabenvielfalt und Ganzheitlichkeit als Gefahr. Neben den Risiken für die Arbeitsgestaltung benennen die Expertengruppen außerdem häufig Risiken für die individuelle Dimension der Mitarbeitenden. Hierbei werden vor allem Risiken für die Gesundheit beschrieben, die sowohl mögliche physische Folgen (z.B. Ermüdung der Augen) als auch psychische Folgen (z. B. Überforderung, Burnout) beinhalten.

Ein Vergleich der Expertengruppen zeigt, dass die Anwender wesentlich seltener Chancen und Risiken für die
Arbeitsgestaltung benennen als die anderen Expertengruppen. Dafür benennen sie wesentlich häufiger Risiken für die technologische Dimension und dabei vor allem bezogen auf die Systemqualität (z.B. Datenschutz, Manipulierbarkeit der DWAS). Weiterhin ist interessant, dass Wissenschaftler sowohl am häufigsten Chancen als auch Risiken der DWAS für die Arbeitsgestaltung benennen. Damit unterscheidet sich diese Expertengruppe von den übrigen Expertengruppen, die die meisten Chancen für die Ökonomie benennen. Um Risiken bei der Anwendung von DWAS in der Produktion vorzubeugen, ist eine bewusste Anwendung und Gestaltung der DWAS notwendig. 

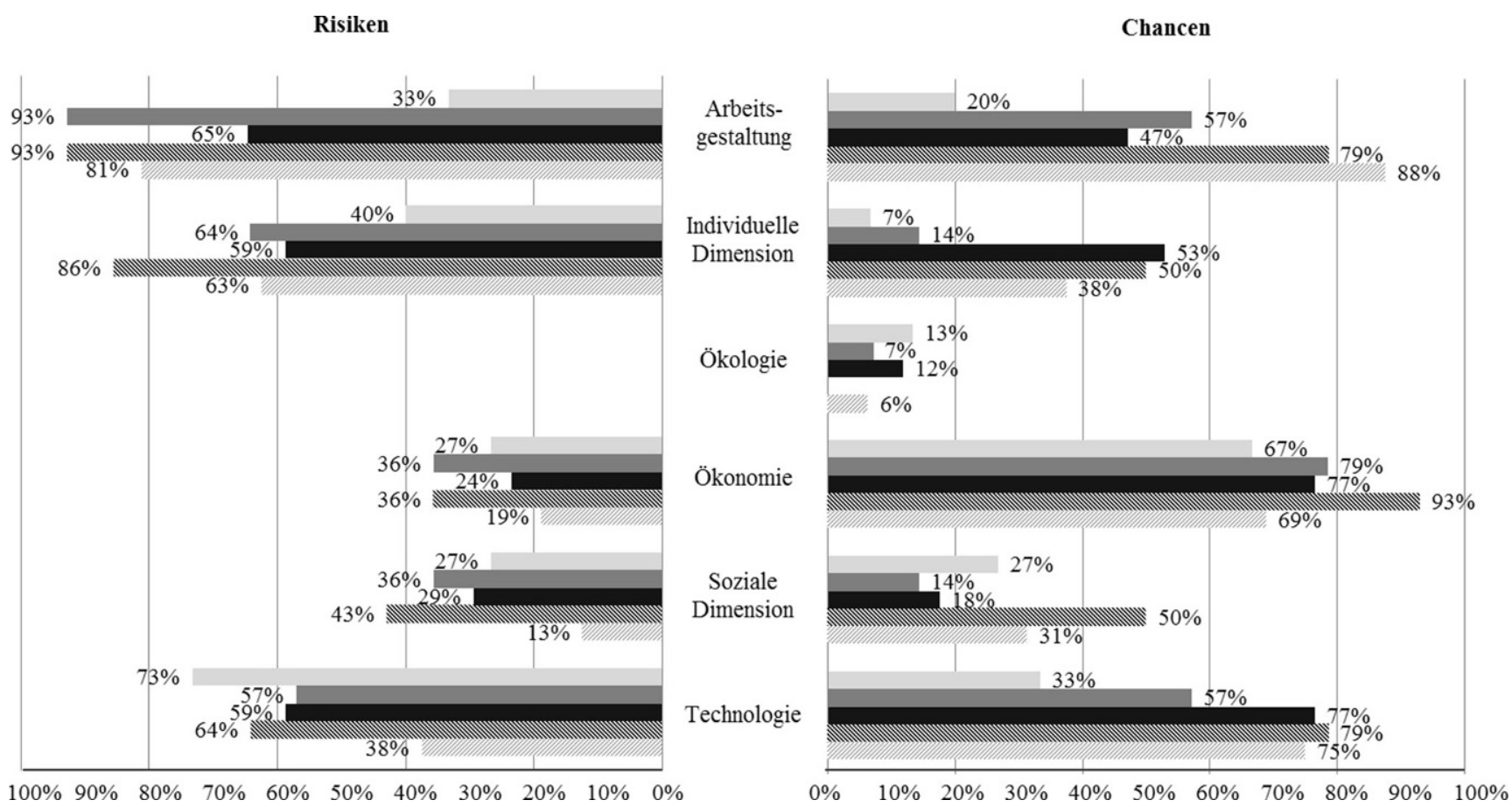

Abb. 5 Prozentuale Nennhäufigkeit der benannten Chancen und Risiken je Expertengruppe

\section{Diskussion}

In diesem Abschnitt werden die Ergebnisse der Studie zusammengefasst und eingeordnet. Danach werden aus den Ergebnissen Gestaltungsempfehlungen für die Anwendung von DWAS in der betrieblichen Praxis abgeleitet und beschrieben.

\subsection{Zusammenfassung und Interpretation der Ergebnisse}

Ziel dieser Studie war es, Anwendungsmöglichkeiten für DWAS sowie Chancen und Risiken beim Einsatz von DWAS im Produktionsumfeld aus der Sicht unterschiedlicher Expertengruppen zu erfassen, um daraus Handlungsund Gestaltungsempfehlungen ableiten zu können. Als Ergebnis wurde ein Kategoriensystem zur Systematisierung von Anwendungsmöglichkeiten von DWAS mit neun Kategorien erstellt.

Das Kategoriensystem kann betriebliche Entscheidungsprozesse im Rahmen des Technologiemanagements unterstützen und bietet einen Überblick über Anwendungsmöglichkeiten und Gestaltungsoptionen. Die Hauptkategorien des Kategoriensystems können von Unternehmen genutzt werden, um einen Überblick über mögliche Anwendungsfälle für DWAS zu erhalten und $\mathrm{zu}$ entscheiden, welche Anwendungsmöglichkeiten in der betrieblichen Praxis realisiert werden sollen. Diese Entscheidungen bilden die Grundlage für die Erstellung eines Technologieplans, in dem neben der konkreten Technologie auch der Zweck der Anwendung festgelegt wird (Schuh et al. 2011). Das Kategoriensystem erweitert bestehende Forschungserkenntnisse durch die Beschreibung von Anwendungsmöglichkeiten, die sich heute im Unternehmensumfeld ggf. noch nicht oder nur schwer realisieren lassen. Ein Beispiel hierfür ist die Nutzung von DWAS zum Arbeitsschutz. DWAS können durch die Anzeige objektbezogener Warn- und Sicherheitshinweise (Niemöller et al. 2017) Mitarbeitende beispielsweise vor Kollisionen warnen. Nützlich ist diese Funktion, wenn sich Mitarbeitende dem Bewegungsraum eines Roboters nähern oder fahrerlose Transportsysteme innerhalb von Fertigungshallen eingesetzt werden. Um diese Anwendung zu realisieren, werden dauerhaft die Positions-, Bewegungs- und Routendaten aller in einer Fertigungshalle befindlichen Maschinen und Fahrzeuge sowie die Standortdaten der Mitarbeitenden benötigt. Für eine zuverlässige objektbezogene Warnung fehlen derzeit häufig noch die notwendigen Sensoren zur Erfassung der benötigten Daten sowie rechtliche und betriebliche Regelungen für deren Erfassung, Auswertung und Verwertung (Roßnagel 2008).

Die Subkategorien des Kategoriensystems „Anwendungsmöglichkeiten“ stellen außerdem unterschiedliche Gestaltungsoptionen bei der Anwendung von DWAS in der betrieblichen Praxis bereit (siehe Tab.2). Beispielsweise kann bei der Anwendung von DWAS zur Unterstützung der Arbeitstätigkeit sowohl die Gestaltungsoption dauerhafte Schritt-für-Schritt-Anleitung als auch die Gestaltungsoption einer bedarfsorientierten temporären Unterstützung der 
Mitarbeitenden realisiert werden. Der Einsatz von DWAS zur dauerhaften Schritt-für-Schritt-Anleitung kann mit Erwartungen wie einer höheren Effizienz der Prozessabläufe und einer höheren Produktqualität verbunden sein, die der Einführung von Technologien im Arbeitsprozess häufig zugrunde liegen (Pfeiffer 2010). Da Mitarbeitende schrittweise durch den Arbeitsprozess geführt werden, können Abweichungen und Fehler verhindert werden. Gleichzeitig kann die detaillierte Vorgabe von Arbeitsschritten von Mitarbeitenden als Einschränkung des Handlungsspielraums und der Autonomie empfunden werden (BAuA 2017). Bestehende Forschungsergebnisse zur Arbeitsgestaltung zeigen, dass Autonomie auf Outcomes wie die Arbeitszufriedenheit (Humphrey et al. 2007; Wu et al. 2015) und die Teamleistung (Cordery et al. 2010) wirkt. Somit könnte eine Verringerung der Autonomie diese Outcomes negativ beeinflussen.

Eine Besonderheit dieser Studie liegt im Einbezug der Sichtweisen unterschiedlicher Expertengruppen und ist unserem Wissen nach die einzige Studie zu DWAS, die sowohl Expertengruppen außerhalb von Unternehmen als auch unterschiedliche Expertengruppen innerhalb von Unternehmen bis hin zu Anwendern berücksichtigt und deren Sichtweisen gegenüberstellt. Der Vergleich der Antworten der Expertengruppen zeigt, dass die betrieblichen Expertengruppen vor allem Anwendungsmöglichkeiten benennen, bei denen die Steigerung der Effizienz und der Qualität im Sinne einer Smart Factory angestrebt werden. Eine mögliche Ursache dafür könnte sein, dass diese Aufgaben bereits heute Bestandteile der Arbeitstätigkeit der Anwender sind und diese auch zukünftig als relevante Bestandteile der Arbeitstätigkeit wahrgenommen werden. Wissenschaftler und politische Akteure benennen im Unterschied zu den anderen Expertengruppen sehr häufig die Anwendung von DWAS für die Qualifizierung und Kompetenzentwicklung. Eine Ursache für den großen Unterschied der Nennhäufigkeiten könnte sein, dass diese Gruppen in der Nutzung von DWAS eine Möglichkeit zur Verbesserung der Arbeit in der Produktion im Sinne einer humanorientierten Arbeitsgestaltung sehen (Wissenschaftlicher Beirat der Plattform Industrie 4.0 2014). Dabei rücken der Erhalt und die Förderung kognitiver Fähigkeiten und eine lernförderliche Gestaltung der Arbeitstätigkeit auch im Sinne des arbeitsintegrierten Lernens stärker in den Fokus. Dafür spricht auch, dass Wissenschaftler und politische Akteure häufig Chancen beim Einsatz von DWAS für die Wissensmerkmale der Tätigkeit benennen. Wissensmerkmale beziehen sich auf die Komplexität der anfallenden Aufgaben, die Anforderungen an Informationsverarbeitung, die Problemlösung, die Anforderungsvielfalt und die Tiefe des erforderlichen Spezialwissens (Morgeson und Humphrey 2006; Stegmann et al. 2010). Bei der bewussten Gestaltung dieser Anwendungsmöglichkeit von DWAS wird es Mitarbeitenden er- möglicht, komplexere und vielfältigere Aufgaben bewältigen oder sich aktiver in die Problemlösung einbringen zu können. Daran ist häufig die Hoffnung einer weiteren Verbesserung und Aufwertung der Produktionsarbeit geknüpft (Richter et al. 2017; Wissenschaftlicher Beirat der Plattform Industrie 4.0 2014).

Um zusätzlich zu den identifizierten Anwendungsmöglichkeiten Gestaltungshinweise ableiten zu können, wurden Chancen und Risiken beim Einsatz von DWAS in der Produktion erfasst. Ergebnisse unserer Studie zeigen, dass der Einsatz von DWAS für die Arbeitsgestaltung ambivalent wahrgenommen wird und sowohl positive als auch negative Veränderungen beschrieben werden. Positive Entwicklungsmöglichkeiten für die Arbeitsgestaltung werden vor allem in der Ausweitung des Tätigkeitsspielraums und dem arbeitsintegrierten Lernen gesehen. Risiken für die Arbeitsgestaltung bestehen darin, dass Tätigkeiten zukünftig weniger Wissen, Fähigkeiten und Problemlösung erfordern, Mitarbeitende daher einer weniger qualifizierten Tätigkeit nachgehen und einfacher ausgetauscht werden können. Erste Studien belegen, dass Unternehmen Digitalisierungslösungen nutzen, um sich vom Fach- und Prozesswissen der Mitarbeitenden unabhängig zu machen (Butollo et al. 2018) und unterstützen das von den Expertengruppen benannte Risiko. Der ambivalente Zusammenhang zwischen Technologieeinführung und Arbeitsgestaltung wurde auch in anderen Studien beschrieben (z. B. Amick und Celentano 1991; Feenberg 1990; Wall et al. 1990) und unterstützt die Forderung nach einer bewussten Gestaltung der Technologien, bei der Auswirkungen auf die Arbeitsgestaltungen bereits ab der Entwicklung der Technologien mitgedacht werden.

Große ökonomische Chancen werden vor allem durch die Steigerungen der Effizienz und der Qualität erwartet. Die Effizienzsteigerungen können vor allem durch die Reduzierung unnötiger Laufwege und die Anleitung der effizientesten Tätigkeitsausführungen erreicht werden. Bei der Qualität wird eine Reduzierung der Fehlerquote beispielsweise durch die automatische Kontrolle des Produkt- bzw. Objektzustandes oder die Bestätigung von Arbeitsschritten erwartet. Die von den Experten benannten ökonomischen Chancen liegen der Einführung von DWAS häufig zugrunde und bilden Kernelemente des Zielbilds der Smart Factory in der Industrie 4.0 (Kagermann et al. 2013).

Anwender benennen vor allem Risiken für die technologische Dimension und hierbei vor allem die Manipulierbarkeit und Störanfälligkeit der Systeme sowie das Thema Datenschutz. Eine mögliche Ursache für die kritische Betrachtung der technologischen Dimension kann sich aus der praktischen Erfahrung der Anwender mit der Einführung neuer Technologien im Unternehmen ergeben. Nehmen Mitarbeitende an, dass der Einsatz neuer Technologien zu einer Verschlechterung ihrer Arbeitsleistung führt, 
werden neue Technologien seltener akzeptiert und eher abgelehnt (Ullrich et al. 2017).

\subsection{Hinweise zur Gestaltung und Nutzung von DWAS}

Aus den identifizierten Chancen und Risiken beim Einsatz von DWAS ergeben sich zahlreiche Hinweise für die Gestaltung und Implementierung der Systeme in der betrieblichen Praxis. Nachfolgend werden einige Gestaltungshinweise für die Arbeitsgestaltung, die individuelle und die technologische Dimension beschrieben.

\subsubsection{Gestaltungsempfehlungen für die Arbeitsgestaltung}

Die Interviewteilnehmer benannten für die Dimension Arbeitsgestaltung vor allem das Risiko, dass durch DWAS Wissensanforderungen an Mitarbeitende sinken, da beispielsweise Arbeitsabläufe und weiterführende Informationen den Mitarbeitenden dauerhaft zur Verfügung gestellt und Tätigkeitsabläufe stärker fragmentiert werden. Auf der anderen Seite wurden ebenfalls große Chancen beim Einsatz von DWAS für eine lernförderliche Gestaltung der Arbeit benannt. Ansatzpunkte bei der lernförderlichen Gestaltung von Arbeit liegen in der Schaffung geeigneter Rahmenbedingungen und der Unterstützung von arbeitsintegrierten Lernprozessen (Mühlbradt et al. 2019).

Die Schaffung lernförderlicher Rahmenbedingungen kann durch den Einsatz von DWAS zur Anleitung der Arbeitstätigkeit unterstützt werden. DWAS liefern den Mitarbeitenden gezielte Hilfestellungen und ermöglichen dadurch die Bewältigung komplexerer Aufgabeninhalte (Anlauft et al. 2019). Dadurch könnten Maßnahmen zur Erweiterung des Handlungs- und Entscheidungsspielraums wie beispielsweise Job Enrichment und Job Enlargement, leichter und mit einem geringeren Qualifizierungsaufwand realisiert werden. Die Anwendung von DWAS zum $M a$ nagement, also zum Erfassen, Verarbeiten und Auswerten von Prozessdaten, kann einen weiteren Ausgangspunkt für die Schaffung lernförderlicher Rahmenbedingungen und die stärkere Integration von Problemlöseprozessen in die Produktionsarbeit darstellen.

Der zweite Ansatzpunkt zur lernförderlichen Gestaltung von Arbeit liegt in der Unterstützung arbeitsintegrierter Lernprozesse. DWAS könnten beispielsweise genutzt werden, um Mitarbeitende individuell und in Abhängigkeit von persönlichen Parametern (Vorerfahrung, Berufserfahrung, gewünschtes Lernformat) beim Erlernen neuer Arbeitstätigkeiten zu unterstützen (Bächler et al. 2019; Klammer et al. 2017). Um Potenziale der DWAS für die Arbeitsgestaltung zu nutzen, sollten Unternehmen bereits bei der geplanten Anwendung von DWAS Möglichkeiten zur Anpassung be- stehender Arbeitsprozesse und -abläufe bedenken und die Integration von Lernmöglichkeiten prüfen.

\subsubsection{Gestaltungsempfehlungen für die individuelle Dimension der Mitarbeitenden}

Für die individuelle Dimension benannten die Expertengruppen häufig Risiken für die physische und psychische Gesundheit. Bisherige Erkenntnisse zeigen, dass durch digitale Technologien Anforderungen an Mitarbeitende steigen und sich beispielsweise Arbeitsmenge, Arbeitsdichte, Zeitdruck, aber auch Arbeitsunterbrechungen und Multitasking erhöhen (Carstensen 2015). Um gesundheitliche Folgen beim Einsatz von DWAS für Mitarbeitende zu minimieren bzw. auszuschließen, sollten Unternehmen zum einen bei der Technologie und zum anderen bei der Schaffung betrieblicher Regelungen ansetzen. Beim Ansatzpunkt der Technologie sollten Unternehmen dafür Sorge tragen, dass die eingesetzten DWAS hinsichtlich gesundheitlicher Folgen fortlaufend evaluiert und im Rahmen der Gefährdungsbeurteilung bewertet werden. Weiterhin kann durch die Schaffung betrieblicher Regelungen der Einsatz und die Nutzung der DWAS zeitlich und örtlich beschränkt werden. Mitarbeitende können so vor negativen gesundheitlichen Folgen wie Stress und Burnout, geschützt werden.

\subsubsection{Gestaltungsempfehlungen für die technologische Dimension}

Für die technologische Dimension wurden in den Interviews große Chancen durch die Verfügbarkeit von Daten und Informationen sowie die Individualisierbarkeit der Informationsdarstellung und die Anpassbarkeit an Nutzerwünsche benannt. Auf der anderen Seite wurden vor allem von Anwendern die Risiken Störanfälligkeit und Manipulierbarkeit der digitalen Technologien sowie das Thema Datenschutz genannt.

Um die Anpassbarkeit der DWAS an Nutzer- und Anwenderbedürfnisse sicherzustellen und die Akzeptanz der Technologien zu fördern, sollten Unternehmen die Perspektive der Anwender frühzeitig in die Gestaltung der Technologien einbeziehen. Einen geeigneten Ansatz hierfür bietet beispielsweise das user centered Design, bei dem zunächst der Nutzungskontext und die Nutzerbedürfnisse erfasst und anschließend passende Lösungen für die Anforderungen der Nutzer erstellt werden. Die Lösungen werden anschließend in einem iterativen Prozess von den Nutzern evaluiert und das Feedback zur Verbesserung und Weiterentwicklung der erstellten Lösung genutzt. Römer und Bruder (2015) haben diese Vorgehensweise bei der Erstellung eines Prototypen für eine Ergonomie-Feedback-App genutzt und beschreiben diese Methode ausführlich. 
Um sensibel auf die Bedenken der Mitarbeitenden beim Thema Datenschutz zu reagieren, sollten Unternehmen frühzeitig betriebliche Reglungen zur Erfassung, Auswertung und Speicherung notwendiger Daten vereinbaren. Der Arbeitnehmerschutz sollte durch die sparsame Erhebung leistungsbezogener Daten und ein Verbot zur personenbezogenen Auswertung sichergestellt werden (Anlauft et al. 2019). Die geltenden Regelungen sollten allen Mitarbeitenden vor der Einführung und Nutzung der DWAS bekannt gemacht werden.

\section{Funding Open Access funding provided by Projekt DEAL.}

Open Access Dieser Artikel wird unter der Creative Commons Namensnennung 4.0 International Lizenz veröffentlicht, welche die Nutzung, Vervielfältigung, Bearbeitung, Verbreitung und Wiedergabe in jeglichem Medium und Format erlaubt, sofern Sie den/die ursprünglichen Autor(en) und die Quelle ordnungsgemäß nennen, einen Link zur Creative Commons Lizenz beifügen und angeben, ob Änderungen vorgenommen wurden.

Die in diesem Artikel enthaltenen Bilder und sonstiges Drittmaterial unterliegen ebenfalls der genannten Creative Commons Lizenz, sofern sich aus der Abbildungslegende nichts anderes ergibt. Sofern das betreffende Material nicht unter der genannten Creative Commons Lizenz steht und die betreffende Handlung nicht nach gesetzlichen Vorschriften erlaubt ist, ist für die oben aufgeführten Weiterverwendungen des Materials die Einwilligung des jeweiligen Rechteinhabers einzuholen.

Weitere Details zur Lizenz entnehmen Sie bitte der Lizenzinformation auf http://creativecommons.org/licenses/by/4.0/deed.de.

\section{Literatur}

Aleksy, M., Rissanen, M. J., Maczey, S., \& Dix, M. (2011). Wearable Computing in Industrial Service Applications. Procedia Computer Science, 5, 394-400.

Amick, B.C., \& Celentano, D.D. (1991). Structural determinants of the psychosocial work environment: introducing technology in the work stress framework. Ergonomics, 34(5), 625-646.

Anlauft, W., Habenicht, T., \& Klippert, J. (2019). Arbeit 4.0 - Proaktive Arbeitsgestaltung als ein zentrales Handlungsfeld für die betriebliche Interessenvertretung. In A. Gerlmaier \& E. Latniak (Hrsg.), Handbuch psycho-soziale Gestaltung digitaler Produktionsarbeit (S. 37-56). Wiesbaden: Springer.

Bächler, A., Bächler, L., Autenrieht, S., Behrendt, H., Funk, M., Krüll, G., Hörz, T., Heidenreich, T., Misselhorn, C., \& Schmidt, A. (2019). Lernförderliche Arbeitsorganisation in der Industrie 4.0. In S. Wischmann \& E. A. Hartmann (Hrsg.), Zukunft der ArbeitEine praxisnahe Betrachtung (S. 195-205). Berlin: Springer.

BAuA (2017). Psychische Gesundheit in der Arbeitswelt - Wissenschaftliche Standortbestimmung. Dortmund: Bundesanstalt für Arbeitsschutz und Arbeitsmedizin. https://www.baua.de/DE/ Angebote/Publikationen/Berichte/Psychische-Gesundheit.pdf? blob=publicationFile. Zugegriffen: 20. Okt. 2019.

Beyer, D., Schiek, M., \& Weissenberger-Eibl, M. A. (2019). Der Weg in die Zukunft. In M. A. Weissenberger-Eibl (Hrsg.), Zukunftsvision Deutschland (Bd. 56, S. 3-13). Heidelberg: Springer.

Brennan, R. L., \& Prediger, D. J. (1981). Coefficient kappa: some uses, misuses, and alternatives. Educational and Psychological Measurement, 41, 687-699.

Brennen, J. S., \& Kreiss, D. (2016). Digitalization. In K. B. Jensen, R. T. Craig, J.D. Pooley \& E. W. Rothenbuhler (Hrsg.), The inter- national encyclopedia of communication theory and philosophy. New York: John Wiley \& Sons.

Brunkow, P., \& Hub, M. (2018). Zur Relevanz der Akzeptanz virtueller Techniken: Entwicklung eines Modells zur multikriteriellen Frühphasenbewertung virtueller Techniken. wt Werkstattstechnik online, 3, 162-168.

Bullinger, H.-J. (1994). Einführung in das Technologiemanagement: Modelle, Methoden, Praxisbeispiele. Stuttgart: Teubner.

Butollo, F., Engel, T., Füchtenkötter, M., Koepp, R., \& Ottaiano, M. (2018). Wie stabil ist der digitale Taylorismus? Störungsbehebung, Prozessverbesserungen und Beschäftigungssystem bei einem Unternehmen des Online-Versandhandels. Arbeits- und Industriesoziologische Studien, 11(2), 143-159.

Carstensen, T. (2015). Neue Anforderungen und Belastungen durch digitale und mobile Technologien. WSI Mitteilungen, 3/2015, 187-193.

Cascio, W.F., \& Montealegre, R. (2016). How technology is changing work and organizations. Annual Review of Organizational Psychology and Organizational Behavior, 3(1), 349-375.

Cordery, J.L., Morrison, D., Wright, B.M., \& Wall, T.D. (2010). The impact of autonomy and task uncertainty on team performance: a longitudinal field study. Journal of Organizational Behavior, 31, 240-258.

Damiani, L., Demartini, M., Guizzi, G., Revetria, R., \& Tonelli, F. (2018). Augmented and virtual reality applications in industrial systems: a qualitative review towards the industry 4.0 era. IFACPapersOnLine, 51(11), 624-630.

Dresing, T., \& Pehl, T. (2018). Praxisbuch, Interview, Transkription und Analyse: Anleitungen und Regelsysteme für qualitativ Forschende (8. Aufl.). Marburg. http://www.audiotranskription.de/ praxisbuch?utm_source=PDF-Praxisbuch\&utm_medium=Text link\&utm_campaign=Verweis-auf-Praxisbuch. Zugegriffen: 29. Jan. 2020.

Evers, M., Krzywdzinski, M., \& Pfeiffer, S. (2018). Designing wearables for use in the workplace: the role of solution developers. Discussion Paper. Berlin. https://bibliothek.wzb.eu/pdf/2018/ iii18-301.pdf. Zugegriffen: 20. Okt. 2019.

Feenberg, A. (1990). The ambivalence of technology. Sociological Perspectives, 33(1), 35-50.

Folkerts, L., \& Hauschildt, J. (2001). Personelle Dynamik in Innovationsprozessen: Neue Fragen und Befunde zum Promotorenmodell. Manuskripte aus den Instituten für Betriebswirtschaftslehre der Universität Kiel, No. 535. Kiel: Universität Kiel, Institut für Betriebswirtschaftslehre.

Froschauer, U., \& Lueger, M. (2003). Das qualitative Interview: Zur Praxis interpretativer Analyse sozialer Systeme (1. Aufl.). UTB Soziologie, Bd. 2418. Wien: WUV.

Hackman, J. R., \& Oldham, G. R. (1976). Motivation through the design of work: test of a theory. Organizational Behavior and $\mathrm{Hu}$ man Performance, 16, 250-279.

Hauschildt, J., \& Chakrabarti, A. K. (1988). Arbeitsteilung im Innovationsmanagement - Forschungsergebnisse, Kriterien und Modelle. Zeitschrift Führung und Organisation, 57, 378-388.

Hauschildt, J., \& Salomo, S. (2008). Promotoren und Opponenten im organisatorischen Umbruch. In R. Fisch, A. Müller \& D. Beck (Hrsg.), Veränderungen in Organisationen - Stand und Perspektiven (S. 163-176). Wiesbaden: Verlag für Sozialwissenschaften.

Hennen, L., Bellucci, S., Berloznik, R., Cope, D., Cruz-Castro, L., Karapiperis, T., Ladikas, M., Klüver, L., Sanz-Menéndez, , Statman, J., Stephan, S., \& Szapiro, T. (2004). Towards a framework for assessing the impact of technology assessment. In M. Decker, M. Ladikas, S. Stephan \& F. Wütscher (Hrsg.), Bridges between science, society and policy (S. 57-85). Berlin: Springer.

Herterich, M.M., Peters, C., Uebernickel, F., Brenner, W., \& Neff, A. A. (2015). Mobile work support for field service: a literature review and directions for future research. In O. Thomas \& F. Teuteberg (Hrsg.), Smart enterprise engineering: 12. Internationa- 
le Tagung Wirtschaftsinformatik (WI 2015): Tagungsband. Osnabrück: Universität Osnabrück.

Hirsch-Kreinsen, H. (2014). Wandel von Produktionsarbeit - „Industrie 4.0“: Arbeitspapier Nr. 38 (Januar 2014). In H. Hirsch-Kreinsen \& J. Weyer (Hrsg.), Soziologisches Arbeitspapier. Dortmund: TU Dortmund.

Hirsch-Kreinsen, H. (2016). Industrie 4.0 als Technologieversprechen: Arbeitspapier Nr. 46 (Juni 2016). In H. Hirsch-Kreinsen, J. Weyer \& M. Wilkesmann (Hrsg.), Soziologisches Arbeitspapier. Dortmund: TU Dortmund.

Howell, J. M., \& Higgins, C. A. (1990). Champions of Technological Innovation. Administrative Science Quarterly, 35(2), 317-341.

Howell, J.M., Shea, C.M., \& Higgins, C. A. (2005). Champions of product innovations: defining, developing, and validating a measure of champion behavior. Journal of Business Venturing, 20, $641-661$.

Humphrey, S. E., Nahrgang, J.D., \& Morgeson, F. P. (2007). Integrating motivational, social, and contextual work design features: a metaanalytic summary and theoretical extension of the work design literature. Journal of Applied Psychology, 92(5), 1332-1356.

Ittermann, P., Niehaus, J., Hirsch-Kreinsen, H., Dregger, J., \& ten Hompel, M. (2016). Social Manufacturing and Logistics: Gestaltung von Arbeit in der digitalen Produktion und Logistik: Soziologisches Arbeitspapier Nr. 47. In H. Hirsch-Kreinsen, J. Weyer \& M. Wilkesmann (Hrsg.), Soziologisches Arbeitspapier. Dortmund: TU Dortmund.

Jørgensen, M. S., Jørgensen, U., \& Clausen, C. (2009). The social shaping approach to technology foresight. Futures, 41(2), 80-86.

Kagermann, H., Wahlster, W., \& Helbig, J. (Hrsg.). (2013). Deutschlands Zukunft als Produktionsstandort sichern - Umsetzungsempfehlungen für das Zukunftsprojekt Industrie 4.0: Abschlussbericht des Arbeitskreises Industrie 4.0. Berlin. https://www.bmbf.de/ files/Umsetzungsempfehlungen_Industrie4_0.pdf. Zugegriffen: 29. Jan. 2020.

Kallio, H., Pietilä, A.M., Johnson, M., \& Kangasniemi, M. (2016). Systematic methodological review: developing a framework for a qualitative semi-structured interview guide. Journal of Advanced Nursing, 72(12), 2954-2965.

Klammer, U., Steffes, S., Maier, M.F., Arnold, D., Stettes, O., Bellmann, L., \& Hirsch-Kreinsen, H. (2017). Arbeiten 4.0 - Folgen der Digitalisierung für die Arbeitswelt. Zeitgespräch. Wirtschaftsdienst, 97(7), 459-476.

MacKenzie, D. A., \& Wajcman, J. (Hrsg.). (1999). The social shaping of technology. Buckingham: Open University Press.

McKinsey Global Institute (2015). The Internet of Things: mapping the value beyond the hype. https://www.mckinsey.com/ $\sim /$ media/McKinsey/Industries/Technology\%20Media\%20and $\% 20$ Telecommunications/High\%20Tech/Our\%20Insights/The $\% 20$ Internet $\% 20$ of $\% 20$ Things $\% 20$ The $\% 20$ value $\% 20$ of $\% 20$ digitizing $\% 20$ the $\% 20$ physical $\% 20$ world/The-Internet-of-thingsMapping-the-value-beyond-the-hype.ashx. Zugegriffen: 2. Jan. 2020.

Morgeson, F. P., \& Campion, M. A. (2003). Work design. In W. C. Borman, D. R. Ilgen \& R.J. Klimoski (Hrsg.), Handbook of psychology:Industrial and organizational psychology (Bd. 12, S. 423-452). Hoboken, New York: Wiley.

Morgeson, F.P., \& Humphrey, S.E. (2006). The Work Design Questionnaire (WDQ): Developing and validating a comprehensive measure for assessing job design and the nature of work. Journal of Applied Psychology, 91(6), 1321-1339.

Mühlbradt, T., Kuhlang, P., \& Finsterbusch, T. (2019). Lernförderliche Arbeitsorganisation in der Industrie 4.0. In S. Wischmann \& E. A. Hartmann (Hrsg.), Zukunft der Arbeit - Eine praxisnahe Betrachtung (S. 195-205). Berlin: Springer.

Niehaus, J. (2017). Mobile Assistenzsysteme für Industrie 4.0: Gestaltungsoptionen zwischen Autonomie und Kontrolle. https://www.
fgw-nrw.de/fileadmin/user_upload/Impuls-I40-04-Niehaus-A1web.pdf. Zugegriffen: 20. Okt. 2019.

Niemöller, C., Zobel, B., Berkemeier, L., Metzger, D., Werning, S., Adelmeyer, T., \& Thomas, O. (2017). Sind Smart Glasses die Zukunft der Digitalisierung von Arbeitsprozessen? Explorative Fallstudien zukünftiger Einsatzszenarien in der Logistik. In J. M. Leimeister \& W. Brenner (Hrsg.), 13. Internationale Tagung Wirtschaftsinformatik (WI 2017). St. Gallen: Institut für Wirtschaftsinformatik St. Gallen.

Okoli, C., \& Pawlowski, S. D. (2004). The Delphi method as a research tool: an example, design considerations and applications. Information \& Management, 42(1), 15-29.

Parker, S. K., \& Grote, G. (2019). Automation, algorithms, and beyond: why work design matters more than ever in A digital world. Applied Psychology. https://doi.org/10.1111/apps.12241.

Parker, S. K., \& Wall, T. D. (2001). Work design: learning from the past and mapping a new terrain. In N. Anderson, D. S. Ones, H. K. Sinangil \& C. Viswesvaran (Hrsg.), Handbook of industrial, work and organizational psychology (S. 90-109). London: SAGE.

Parker, S. K., Wall, T. D., \& Cordery, J.L. (2001). Future work design research and practice: towards an elaborated model of work design. Journal of Occupational and Organizational Psychology, $74,413-440$

Pfeiffer, S. (2010). Technisierung von Arbeit. In F. Böhle, G. G. Voß \& G. Wachtler (Hrsg.), Handbuch Arbeitssoziologie (S. 231-261). Wiesbaden: VS.

Qu, S., \& Dumay, J. (2011). The qualitative research interview. Qualitative Research in Accounting \& Management, 8(3), 238-264.

Rädiker, S., \& Kuckartz, U. (2019). Analyse qualitativer Daten mit $M A X Q D A$. Wiesbaden: Springer.

Rammert, W. (1993). Technik aus soziologischer Perspektive. Wiesbaden: Springer.

Rammert, W. (2016). Technik - Handeln - Wissen. Wiesbaden: Springer.

Richter, A., Heinrich, P., Stocker, A., \& Steinhüser, M. (2017). Die neue Rolle des Mitarbeiters in der digitalen Fabrik der Zukunft. In S. Reinheimer (Hrsg.), Industrie 4.0 (S. 91-115). Wiesbaden: Springer.

Römer, T., \& Bruder, R. (2015). User centered design of a cyber-physical support solution for assembly processes. Procedia Manufacturing, 3, 456-463.

Roßnagel, A. (2008). Selbst- oder Fremdbestimmung - Die Zukunft des Datenschutzes. In A. Roßnagel, T. Sommerlatte \& U. Winand (Hrsg.), Digitale Visionen (S. 123-163). Heidelberg: Springer.

Russell, S., \& Williams, R. (2002). Social shaping of technology: Frameworks, Findings and Implications for policy: with glossary of social shaping concepts. In K. H. Sørensen \& R. Williams (Hrsg.), Shaping technology, guiding policy: concepts, spaces and tools (S. 35-112). Cheltenham: Edward Elgar.

Schaper, N. (2019). Arbeitsgestaltung in Produktion und Verwaltung. In F. W. Nerdinger, G. Blickle \& N. Schaper (Hrsg.), Arbeits- und Organisationspsychologie (S. 411-434). Berlin: Springer.

Schuh, G., Klappert, S., \& Moll, T. (2011). Ordnungsrahmen Technologiemanagement. In G. Schuh \& S. Klappert (Hrsg.), Technologiemanagement (Bd. 29, S. 11-31). Heidelberg: Springer.

Spath, D., Ganschar, O., Gerlach, S., Hämmerle, M., Krause, T., \& Schlund, S. (2013). Produktionsarbeit der Zukunft - Industrie 4.0: Studie. Stuttgart. http://www.mechatronik-bw.de/attachments/ article/272/Fraunhofer-IAO-Studie_Produktionsarbeit_der_ Zukunft_-_Industrie_4.0.pdf. Zugegriffen: 20. Okt. 2019.

Stegmann, S., van Dick, R., Ullrich, J., Charalambous, J., Menzel, B., Egold, N., \& Wu, T.T.-C. (2010). Der Work Design Questionnaire. Zeitschrift für Arbeits- und Organisationspsychologie, $54(1), 1-28$.

Steigleder, S. (2008). Die strukturierende qualitative Inhaltsanalyse im Praxistest. Eine konstruktive kritische Studie zur Auswertungsmethodik von Philipp Mayring. Marburg: Tectum. 
Stolterman, E., \& Fors, C. A. (2004). Information technology and the good life. In B. Kaplan, D. P. Truex III, D. Wastell, A. T. WoodHarper \& J.I. DeGross (Hrsg.), Information systems research - relevant theory and practice (S. 687-692). Boston: Springer.

Trist, E. (1981). The socio-technical perspective: The evolution of sociotechnical systems as a conceptual framework and as an action research program. In A.H.W. F. J. Van de Ven (Hrsg.), Perspectives on organization design and behavior (S. 17-75). New York: Wiley.

Trist, E., \& Bamforth, K.W. (1951). Some social and psychological consequences of the longwall method of coal-getting. Human Relation, 4(1), 3-38.

Ullrich, A., Thim, C., Vladova, G., \& Gronau, N. (2017). Wandlungsbereitschaft und Wandlungsfähigkeit von Mitarbeitern bei der Transformation zu Industrie 4.0. In S. Reinheimer (Hrsg.), Industrie 4.0 (S. 91-115). Wiesbaden: Springer.

VDI - Verein Deutscher Ingenieure (2000). Technikbewertung - Begriffe und Grundlagen. (VDI-Richtlinie, 3780). Düsseldorf: VDI.

VERBI Software (2017). MAXQDA 2018 [Computer Programm]. Berlin: Sozialforschung. https://www.maxqda.com

Wall, T.D., Corbett, J.M., Martin, R., Clegg, C.W., \& Jackson, P.R. (1990). Advanced manufacturing technology, work design, and performance: a change study. Journal of Applied Psychology, 75(6), 691-697.

Waschull, S., Bokhorst, J.A.C., Molleman, E., \& Wormann, J.C. (2020). Work design in the future industrial production: transforming towards cyber-physical systems. Computers \& Industrial Engineering. https://doi.org/10.1016/j.cie.2019.01.053.

Wieseke, J., Kraus, F., \& Rajab, T. (2010). Ein interdisziplinärer Ansatz zur Überwindung von Technologieadaptionsbarrieren. Zeitschrift für betriebswirtschaftliche Forschung, 62(7), 822-859.

Williams, R., \& Edge, D. (1996). The social shaping of technology. Research Policy, 25(6), 865-899.

Wissenschaftlicher Beirat der Plattform Industrie 4.0. (2014). Neue Chancen für unsere Produktion. 17 Thesen des Wissenschaftlichen Beirats der Plattform Industrie 4.0. Berlin. https://www.itsowl.de/fileadmin/PDF/Industrie_4.0/Thesen_des_wissenschaftli chen_Beirats_Industrie_4.0.pdf. Zugegriffen 31. Okt. 2019

Witte, E. (1973). Organisation für Innovationsentscheidungen: Das Promotoren-Modell. Göttingen: Schwartz.

Wixom, B.H., \& Todd, P. A. (2005). A theoretical integration of user satisfaction and technology acceptance. Information Systems Research, 16(1), 85-102.

Wu, C.-H., Griffin, M. A., \& Parker, S. K. (2015). Developing agency through good work: longitudinal effects of job autonomy and skill utilization on locus of control. Journal of Vocational Behavior, 89, 102-108.

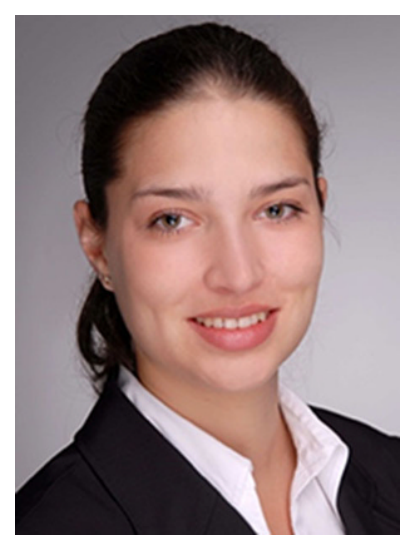

Verena Simone Lore Blumberg promoviert seit 2015 in Kooperation mit einem Mobilitätsunternehmen und wird von Frau Prof. Kauffeld am Lehrstuhl für Arbeits-, Organisations- und Sozialpsychologie der Technischen Universität Braunschweig betreut. Ihr Forschungsschwerpunkt ist die Veränderung von Arbeit durch die Einführung neuer Technologien.

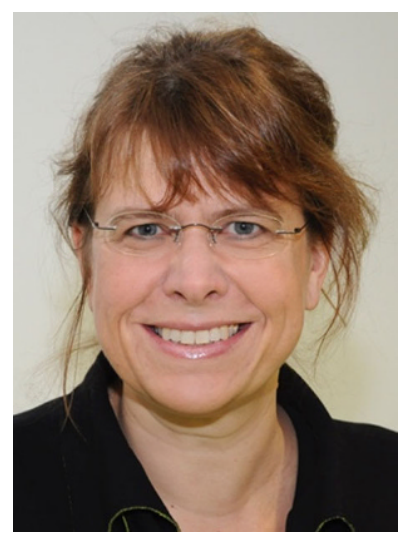

Prof. Dr. Simone Kauffeld ist Inhaberin des Lehrstuhls für Arbeit-, Organisations- und Sozialpsychologie der Technischen Universität Braunschweig. In ihrer Forschungstätigkeit setzt sie sich in zahlreichen Projekten mit den Themen Kompetenzentwicklung und -management (Training und Transfer), Team und Führung, Karriere/Coaching sowie Veränderungen in Organisation und Arbeit auseinander. Das Thema Digitalisierung ist als Querschnittsthema präsent. Als Herausgeberin hat sie die Zeitschriften „PersonalQUARTERLY“ und „Gruppe. Interaktion. Organisation" neu aufgesetzt und gibt Buchreihen zur Arbeits- und Organisationspsychologie heraus. Um aktiven Wissenstransfer zu leisten, hat sie 2008 die 4A-SIDE GmbH gegründet, die psychologische Expertise mit IT-Kompetenz verbindet. 